MPP-2014-50

\title{
Ninja: Automated Integrand Reduction via Laurent Expansion for One-Loop Amplitudes
}

\author{
Tiziano Peraro \\ Max-Planck Insitut für Physik, \\ Föhringer Ring, 6, D-80805 München, Germany \\ E-mail: peraro@mppmu.mpg.de
}

\begin{abstract}
We present the public $\mathrm{C}++$ library NINJA, which implements the Integrand Reduction via Laurent Expansion method for the computation of one-loop integrals. The algorithm is suited for applications to complex one-loop processes.
\end{abstract}




\section{Contents}

1 Introduction $\quad 2$

2 Integrand Reduction via Laurent Expansion 4

2.1 Universal one-loop decomposition . . . . . . . . . . . . . . . . . . . 4

2.2 The Laurent expansion method . . . . . . . . . . . . . . . . . 7

3 Semi-numerical implementation $\quad 12$

3.1 Input . . . . . . . . . . . . . . . . . . . . . . . 12

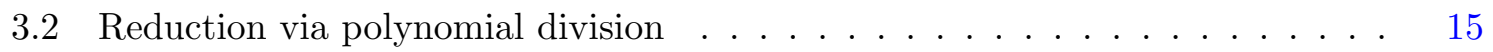

3.3 Master Integrals . . . . . . . . . . . . . . . . . . . . . . . 16

4 Basic usage $\quad 16$

4.1 Installation . . . . . . . . . . . . . . . . . . . 16

4.2 Writing the Integrand $\ldots \ldots \ldots \ldots \ldots \ldots$

4.3 Running the reduction . . . . . . . . . . . . . . . . . . . . 19

4.3 .1 A simple example . . . . . . . . . . . . . . . 20

4.3 .2 The Amplitude class . . . . . . . . . . . . . . . . . 22

4.3 .3 Global settings . . . . . . . . . . . . . . . . 25

5 Examples $\quad 28$

5.1 Simple Test . . . . . . . . . . . . . . . . . . . . . . . . . . . . . . . . . . .

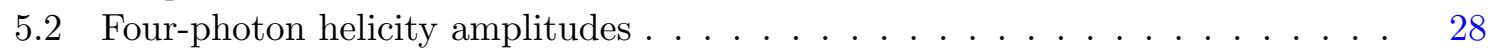

5.3 Six-photon helicity amplitudes . . . . . . . . . . . . . . . . 30

5.4 Five-point diagram of $g g \rightarrow H t \bar{t} \ldots \ldots \ldots \ldots \ldots \ldots \ldots$

5.5 Higher-rank example . . . . . . . . . . . . . . . . . . . . . . . . . . . . . . . . . . . .

5.6 Usage in multi-threaded applications . . . . . . . . . . . . . . 33

6 Conclusions $\quad 35$

$\begin{array}{lll}\text { Appendix A } & \text { The Python package NinjaNumGen } & 36\end{array}$

$\begin{array}{ll}\text { Appendix B Higher-rank numerators } & 38\end{array}$

B.1 Reduction of higher-rank integrands . . . . . . . . . . . . . . . . . . . . . . . . . . . . . .

B.2 The input . . . . . . . . . . . . . . . . . . . . . . 40

B.3 Higher-rank Master Integrals . . . . . . . . . . . . . . . . . . 40

$\begin{array}{ll}\text { Appendix C Interfaces to Integral Libraries } & 41\end{array}$

C.1 Built-in interfaces . . . . . . . . . . . . . . . . . . . 42

\section{Introduction}

The investigation of the factorization properties of scattering amplitudes at their singularities [1-5] lead to important results in Quantum Field Theory and Theoretical Particle Physics, such as the development of new methods for phenomenological computations. In particular, integrand reduction methods, developed for one-loop diagrams $[6,7]$ and recently extended 
to higher loops [8-12], use the knowledge of the analytic and algebraic structure of loop integrands in order to rewrite scattering amplitudes as linear combinations of Master Integrals.

At one loop, integrand-reduction methods allow to express any integrand in dimensional regularization as a sum of contributions with at most five propagators in the loop, regardless of the number of external legs of the amplitude. The numerators of these contributions are polynomial residues which have a universal parametric form that does not depend on the process. This parametric form can be written as a sum of monomials in the components of the loop momentum, multiplied by unknown process-dependent coefficients. After integration, the amplitude becomes a linear combination of known integrals. The coefficients of this linear combination can be identified with a subset of the ones which parametrize the residues. Therefore, the problem of the computation of any one-loop amplitude can be reduced to the one of performing a polynomial fit of the coefficients of the residues.

The fit of the unknown coefficients can be efficiently performed by evaluating the numerator of the integrand on multiple cuts, i.e. on values of the loop momentum such that a subset of the loop denominators vanish. The multiple-cut conditions can be viewed as projectors which isolate the residue sitting on the cut denominators. A residue can be evaluated by putting on-shell the corresponding loop propagators and subtracting from the integrand the non-vanishing contributions coming from higher-point residues. This leads to a top-down algorithm which allows to compute any one-loop amplitude with any number of external legs.

Within semi-numerical computations, the algorithm is usually implemented by sampling the integrand on several solutions of the multiple cuts and subtracting at each step of the reduction all the non-vanishing contributions coming from higher-point residues. This yields a system of equations for the coefficients of each residue. The method is suited for automation and it has been implemented in several codes, some of which are public (e.g. CuTTools [13] and SAMURAI [14]). Its usage within several automated frameworks [15-23] has been particularly successful and produced highly non-trivial phenomenological results.

In this paper we present a new public $\mathrm{C}++$ library called NinJA, which implements an alternative integrand-reduction algorithm first proposed in Ref. [24]. This is based on the systematic application of the Laurent series expansion to an integrand on the multiple cuts. After performing a suitable Laurent expansion on a multiple cut, in the asymptotic limit both the integrand and the subtraction terms exhibit the same polynomial behavior as the residue. This allows one to directly identify the coefficients of the residues (and thus the ones of the Master Integrals) with the ones of the Laurent expansion of the integrand, corrected by subtractions terms which can be computed once and for all as functions of a subset of the higher-point coefficients. This leads to a diagonal system of equations for each residue and to a significant reduction of the number of subtraction terms which affect the computation of lower-point contributions.

NinJA takes as input the numerator cast into four different forms. The first is a procedure which evaluates it as a function of the loop momentum. The others instead compute the leading terms of properly defined Laurent expansions of the numerator. Since the integrand of a one-loop amplitude is a rational function of the loop momentum, the Laurent expansions for an integrand can be obtained via a partial fraction decomposition. NinJA implements it semi-numerically via a simplified polynomial division algorithm between the expansions of the numerator and the ones of the denominators. The coefficients of the Laurent expansion are then corrected by the subtraction terms and multiplied by the Master Integrals. These are computed by interfacing NINJA with an external library which can be specified by the user. Interfaces for ONELOOP [16,25] and LoOpTools [15] are already provided with the 
distribution.

The simplified subtractions and the diagonal systems of equations make the algorithm implemented in NINJA significantly simpler and lighter than the traditional one. The library has been interfaced with the one-loop package GoSAm [20] and has already been used to compute NLO corrections to Higgs boson production in association with a top-quark pair and a jet [26] and several six-, seven- and eight-point amplitudes involving both massive and massless particles as external states or propagating in the loop [27]. These applications showed that NiNJA has better performance and numerical stability than implementations of traditional integrand reduction algorithms. In particular, Ref. [27] provides a detailed analysis of the performance and accuracy of this library. With this paper, we make Ninja publicly available as a standalone library which can be interfaced to other packages and frameworks for one-loop computations.

In order to simplify the generation of the numerator expansions and the corresponding source code needed by NINJA as input, we distribute with the library a small PYTHON package called NinjaNumGen which uses Form-4 [28-30] in order to compute the expansions and produce an optimized source code which can be used by the library.

In this paper, besides the description of the implementation of the algorithm and the usage of the library, we also propose a method for the extension of the integrand reduction via Laurent expansion for the computation of integrals whose rank is one unit higher than the number of loop denominators. This method is not present elsewhere in the literature and has been implemented in NiNJA, allowing thus to use the library for computations in non-renormalizable and effective theories as well.

The paper is organized as follows. In Section 2 we give a review of the Laurent Expansion method for the integrand reduction of one-loop amplitudes. In Section 3 we discuss its seminumerical implementation in the library NinJA. The usage of the library is explained, with the help of simple examples, in Section 4. In Section 5 we give a description of the examples which are distributed with the library, giving a better view of its usage and capabilities. Appendix A gives more details on the usage of the package NinjaNumGen. In Appendix B we present the extension of the algorithm to higher-rank integrals. Appendix $\mathrm{C}$ gives more information on the interface between NINJA and the libraries of Master Integrals.

Since this paper is rather technical, the reader which is mostly interested in the usage of the library might want to read Sections 4 and 5 first, referring to the previous sections or the appendices at a later time if needed.

\section{Integrand Reduction via Laurent Expansion}

In this section we review the Integrand Reduction via Laurent-expansion method [24] for the computation of one-loop integrals. The method is based on the systematic application of the Laurent series expansion on the universal integrand decomposition of one-loop amplitudes, which allows to reduce any amplitude as a linear combination of known Master Integrals.

\subsection{Universal one-loop decomposition}

A generic contribution $\mathcal{M}$ to an $n$-point one-loop amplitude in dimensional regularization has the form

$$
\mathcal{M}=h\left(\mu_{R}^{2}, d\right) \int d^{d} \bar{q} \mathcal{I}=h\left(\mu_{R}^{2}, d\right) \int d^{d} \bar{q} \frac{\mathcal{N}(\bar{q})}{D_{0} \cdots D_{n-1}} .
$$


In the previous equation, the integrand $\mathcal{I}$ is a rational function of the components of the $d$ dimensional loop momentum $\bar{q}$, with $d=4-2 \epsilon$. The numerator $\mathcal{N}(\bar{q})$ is a process-dependent polynomial in $\bar{q}$, while the denominators $D_{i}$ are quadratic polynomials in $\bar{q}$ and correspond to Feynman loop propagators,

$$
D_{i}=\left(\bar{q}+p_{i}\right)^{2}-m_{i}^{2} .
$$

The function $h$ appearing in Eq. (1) is a conventional normalization factor given by [31]

$$
h\left(\mu_{R}^{2}, d\right)=h\left(\mu_{R}^{2}, 4-2 \epsilon\right)=\frac{\mu_{R}^{2 \epsilon}}{i \pi^{2-\epsilon}} \frac{\Gamma(1-2 \epsilon)}{\Gamma^{2}(1-\epsilon) \Gamma(1+\epsilon)},
$$

as a function of the renormalization scale $\mu_{R}^{2}$ and the dimension $d=4-2 \epsilon$. The $d$-dimensional loop momentum $\bar{q}$ can be split into a four-dimensional part $q$ and a $(-2 \epsilon)$-dimensional part $\vec{\mu}$ as

$$
\bar{q}=q+\vec{\mu}, \quad \bar{q}^{2}=q^{2}-\mu^{2} .
$$

The numerator $\mathcal{N}$ will therefore be a polynomial in the four components of $q$ and the extradimensional variable $\mu^{2}$.

Every one-loop integrand in dimensional regularization can be decomposed as sum of integrands having five or less loop denominators $[6,7]$

$$
\mathcal{I} \equiv \frac{\mathcal{N}}{D_{0} \cdots D_{n-1}}=\sum_{k=1}^{5} \sum_{\left\{i_{1}, \cdots, i_{k}\right\}} \frac{\Delta_{i_{1} \cdots i_{k}}}{D_{i_{1}} \cdots D_{i_{k}}},
$$

where the second sum on the r.h.s. runs over all the subsets of the denominator indexes $\{0, \ldots, n-1\}$ containing $k$ elements. The residues $\Delta_{i_{1} \cdots i_{k}}$ appearing in Eq. (5) are irreducible polynomials, i.e. polynomials which do not contain terms proportional to the corresponding loop denominators $D_{i_{1}}, \ldots, D_{i_{k}}$. These residues have a universal, process-independent parametric form in terms of unknown, process-dependent coefficients.

For any set of denominators $D_{i_{1}}, \ldots, D_{i_{k}}$ with $k \leq 5$, one can build a 4 -dimensional basis of massless momenta $\mathcal{E}^{\left(i_{1} \cdots i_{k}\right)}=\left\{e_{1}, e_{2}, e_{3}, e_{4}\right\}[6,14,32,33]$. The first two elements of the basis are linear combinations of two external momenta $K_{1}, K_{2}$ of the sub-diagram identified by the considered set of loop denominators. More explicitly, we define

$$
e_{1}^{\mu}=\frac{1}{1-r_{1} r_{2}}\left(K_{1}^{\mu}-r_{1} K_{2}^{\mu}\right), \quad e_{2}^{\mu}=\frac{1}{1-r_{1} r_{2}}\left(K_{2}^{\mu}-r_{2} K_{1}^{\mu}\right),
$$

with

$$
\begin{aligned}
& K_{1}^{\mu}=p_{i_{1}}^{\mu}-p_{i_{k}}^{\mu}, \quad K_{2}^{\mu}=p_{i_{2}}^{\mu}-p_{i_{1}}^{\mu}, \quad r_{1}=\frac{K_{1}^{2}}{\gamma}, \quad r_{2}=\frac{K_{2}^{2}}{\gamma}, \\
& \gamma=\left(K_{1} \cdot K_{2}\right)\left(1+\sqrt{1-\frac{K_{1}^{2} K_{2}^{2}}{\left(K_{1} \cdot K_{2}\right)^{2}}}\right)
\end{aligned}
$$

where the momenta $p_{i}$ were defined in Eq. (2). If the sub-diagram has less than two independent external momenta, the remaining ones are substituted by arbitrary reference vectors in the definition of $e_{1}$ and $e_{2}$. The momenta $e_{3}$ and $e_{4}$ are instead chosen to be orthogonal to the first two and can be defined using the spinor notation as

$$
e_{3}^{\mu}=\frac{\left\langle e_{1} \gamma^{\mu} e_{2}\right]}{2}, \quad e_{4}^{\mu}=\frac{\left\langle e_{2} \gamma^{\mu} e_{1}\right]}{2} .
$$


They satisfy the property $\left(e_{3} \cdot e_{4}\right)=-\left(e_{1} \cdot e_{2}\right)$. For subsets of $k=4$ denominators, we also define the vectors $v$ and $v_{\perp}$

$$
v^{\mu}=\left(e_{4} \cdot K_{3}\right) e_{3}^{\mu}+\left(e_{3} \cdot K_{3}\right) e_{4}^{\mu}, \quad v_{\perp}^{\mu}=\left(e_{4} \cdot K_{3}\right) e_{3}^{\mu}-\left(e_{3} \cdot K_{3}\right) e_{4}^{\mu},
$$

with $K_{3}^{\mu}=p_{i_{3}}^{\mu}-p_{i_{2}}^{\mu}$. We observe that the vector $v_{\perp}$ is orthogonal to all the external legs of the sub-diagram identified by the four denominators.

By expanding the four dimensional part $q$ of the loop momentum $\bar{q}$ in the basis $\mathcal{E}^{\left(i_{1} \cdots i_{k}\right)}$, the numerator and the denominators can be written as polynomials in the coordinates $\mathbf{z} \equiv$ $\left(z_{1}, z_{2}, z_{3}, z_{4}, z_{5}\right)=\left(x_{1}, x_{2}, x_{3}, x_{4}, \mu^{2}\right)$,

$$
\mathcal{N}(\bar{q})=\mathcal{N}\left(q, \mu^{2}\right)=\mathcal{N}\left(x_{1}, x_{2}, x_{3}, x_{4}, \mu^{2}\right)=\mathcal{N}(\mathbf{z})
$$

with

$$
q^{\nu}=-p_{i_{1}}^{\nu}+\frac{1}{e_{1} \cdot e_{2}}\left(x_{1} e_{1}^{\nu}+x_{2} e_{2}^{\nu}-x_{3} e_{3}^{\nu}-x_{4} e_{4}^{\nu}\right) .
$$

The coordinates $x_{i}$ can be written as scalar products

$$
x_{1}=\left(l_{i_{1}} \cdot e_{2}\right), \quad x_{2}=\left(l_{i_{1}} \cdot e_{1}\right), \quad x_{3}=\left(l_{i_{1}} \cdot e_{4}\right), \quad x_{4}=\left(l_{i_{1}} \cdot e_{3}\right),
$$

where $l_{i_{1}} \equiv\left(q+p_{i_{1}}\right)$. For $k=4$ we also consider the alternative expansion of the loop momentum

$$
q^{\nu}=-p_{i_{1}}^{\nu}+\frac{1}{e_{1} \cdot e_{2}}\left(x_{1} e_{1}^{\nu}+x_{2} e_{2}^{\nu}\right)+\frac{1}{v^{2}}\left(x_{3, v} v_{3}^{\nu}-x_{4, v} v_{4}^{\nu}\right) .
$$

with

$$
x_{3, v}=\left(l_{i_{1}} \cdot v\right), \quad x_{4, v}=\left(l_{i_{1}} \cdot v_{\perp}\right) .
$$

The universal parametric form of the residues $\Delta_{i_{1} \cdots i_{k}}$ in a renormalizable theory is $[6,7,11]$

$$
\begin{aligned}
\Delta_{i_{1} i_{2} i_{3} i_{4} i_{5}} & =c_{0} \mu^{2} \\
\Delta_{i_{1} i_{2} i_{3} i_{4}} & =c_{0}+c_{1} x_{4, v}+\mu^{2}\left(c_{2}+c_{3} x_{4, v}+\mu^{2} c_{4}\right) \\
\Delta_{i_{1} i_{2} i_{3}} & =c_{0}+c_{1} x_{4}+c_{2} x_{4}^{2}+c_{3} x_{4}^{3}+c_{4} x_{3}+c_{5} x_{3}^{2}+c_{6} x_{3}^{3}+\mu^{2}\left(c_{7}+c_{8} x_{4}+c_{9} x_{3}\right) \\
\Delta_{i_{1} i_{2}} & =c_{0}+c_{1} x_{1}+c_{2} x_{2}^{2}+c_{3} x_{4}+c_{4} x_{4}^{2}+c_{5} x_{3}+c_{6} x_{3}^{2}+c_{7} x_{1} x_{4}+c_{8} x_{1} x_{3}+c_{9} \mu^{2} \\
\Delta_{i_{1}} & =c_{0}+c_{1} x_{2}+c_{2} x_{1}+c_{3} x_{4}+c_{4} x_{3} .
\end{aligned}
$$

where we understand that the unknown coefficients $c_{j}$ depend on the indexes of the residue (e.g. $c_{j}=c_{j}^{\left(i_{1} \cdots i_{k}\right)}$ ), while the scalar products $x_{i}$ and $x_{i, v}$ depend on both the indexes of the residue and the loop momentum $q$. The parametrization in Eq. (14) can be extended to effective and non-renormalizable theories where the rank of the numerator can be larger than the number of loop propagators [24]. More details on the higher-rank case are given in Appendix B.

Most of the terms appearing in Eq. (14) are spurious, i.e. they vanish after integration and do not contribute to the final result. The amplitude $\mathcal{M}$ can thus be expressed as a linear combination of Master Integrals, corresponding to the non-spurious terms of the integrand 
decomposition, namely

$$
\begin{aligned}
\mathcal{M}= & \sum_{\left\{i_{1}, i_{2}, i_{3}, i_{4}\right\}}\left\{c_{0}^{\left(i_{1} i_{2} i_{3} i_{4}\right)} I_{i_{1} i_{2} i_{3} i_{4}}+c_{4}^{\left(i_{1} i_{2} i_{3} i_{4}\right)} I_{i_{1} i_{2} i_{3} i_{4}}\left[\mu^{4}\right]\right\} \\
& +\sum_{\left\{i_{1}, i_{2}, i_{3}\right\}}\left\{c_{0}^{\left(i_{1} i_{2} i_{3}\right)} I_{i_{1} i_{2} i_{3}}+c_{7}^{\left(i_{1} i_{2} i_{3}\right)} I_{i_{1} i_{2} i_{3}}\left[\mu^{2}\right]\right\} \\
& +\sum_{\left\{i_{1}, i_{2}\right\}}\left\{c_{0}^{\left(i_{1} i_{2}\right)} I_{i_{1} i_{2}}+c_{1}^{\left(i_{1} i_{2}\right)} I_{i_{1} i_{2}}\left[\left(q+p_{i_{1}}\right) \cdot e_{2}\right]\right. \\
& \left.+c_{2}^{\left(i_{1} i_{2}\right)} I_{i_{1} i_{2}}\left[\left(\left(q+p_{i_{1}}\right) \cdot e_{2}\right)^{2}\right]+c_{9}^{\left(i_{1} i_{2}\right)} I_{i_{1} i_{2}}\left[\mu^{2}\right]\right\} \\
& +\sum_{i_{1}} c_{0}^{\left(i_{1}\right)} I_{i_{1}},
\end{aligned}
$$

where

$$
I_{i_{1} \cdots i_{k}}[\alpha] \equiv h\left(\mu_{R}^{2}, d\right) \int d^{d} \bar{q} \frac{\alpha}{D_{i_{1}} \cdots D_{i_{k}}}, \quad I_{i_{1} \cdots i_{k}} \equiv I_{i_{1} \cdots i_{k}}[1] .
$$

The coefficients of this linear combination can be identified with a subset of the coefficients of the parametric residues in Eq. (14). Since all the Master Integrals appearing in Eq. (15) are known, the problem of the computation of an arbitrary one-loop amplitude can be reduced to the problem of the determination of the non-spurious coefficients appearing in the parametrization of the residues $\Delta_{i_{1} \cdots i_{k}}$.

\subsection{The Laurent expansion method}

The coefficients appearing in the integrand decomposition can be computed by evaluating the integrand on multiple cuts, i.e. on values of the loop momentum $\bar{q}$ such that a subset of loop denominators vanish [13]. More in detail, the coefficients of a $k$-point residue $\Delta_{i_{1} \cdots i_{k}}$ can be determined by evaluating the integrand on the corresponding $k$-ple cut $D_{i_{1}}=\cdots=D_{i_{k}}=0$. For these values of the loop momentum, the only non-vanishing contributions of the integrand decomposition are the ones coming from the residue in consideration and from all the higherpoint residues which have $\left\{D_{i_{1}}, \ldots, D_{i_{k}}\right\}$ as a subset of their loop denominators.

Within the original integrand reduction algorithm [13,14,34], the coefficients are computed by sampling the numerator of the integrand on a finite subset of the on-shell solutions, subtracting all the non-vanishing contributions coming from higher-point residues, and finally solving the resulting linear system of equations. This is therefore a top-down approach, where higher-point residues are computed first, starting from $k=5$, and systematically subtracted from the integrand for the evaluation of lower-point contributions. These are referred to as subtractions at the integrand level.

The integrand reduction via Laurent expansion method, presented in Ref. [24], improves this reduction strategy by elaborating on techniques proposed in $[32,35]$. Whenever the analytic dependence of the integrand on the loop momentum is known, this approach allows to compute the coefficients of a residue $\Delta_{i_{1} \cdots i_{k}}$ by performing a Laurent expansion of the integrand with respect to one of the components of the loop momentum which are not fixed by the on-shell conditions of the corresponding multiple cut $D_{i_{1}}=\cdots=D_{i_{k}}=0$. In the asymptotic limit defined by this Laurent expansion, both the integrand and the higher-point 
subtractions exhibit the same polynomial behavior as the residue. Therefore one can directly identify the unknown coefficients with the ones of the Laurent expansion of the integrand, corrected by the contributions coming from higher-point residues.

Hence, by choosing a suitable Laurent expansion, one obtains a diagonal system of equations for the coefficients of the residues, while the subtractions of higher-point contributions can be implemented as corrections at the coefficient level which replace the subtractions at the integrand level of the original algorithm. Since the polynomial structure of the residues is universal and does not depend on the process, the parametric form of the coefficient-level corrections can be computed once and for all, in terms of a subset of the higher-point coefficients. More in detail, the corrections at the coefficient level are known functions of a subset of the coefficients of 3- and 2-point residues. In particular, no subtraction term coming from 4- and 5 -point contributions is ever needed. This allows to skip the computation of the (spurious) 5-point contributions entirely, and to completely disentangle the determination of 4-point residues from the one of lower point contributions.

In the following, we address more in detail the computation of 5-, 4-, 3-, 2-, and 1point residues, also commonly known as pentagons, boxes, triangles, bubbles and tadpoles respectively. For simplicity, we focus on renormalizable theories, where (up to a suitable choice of gauge) the maximum allowed rank of the integrand is equal to the number of loop denominators and the most general parametrization of the residues is the one given in Eq. (14). NinJA can also be used for the computation of integrals whose rank exceeds the number of denominators by one. The extension of the method to the higher-rank case is discussed in Appendix B.

5-point residues As mentioned above, pentagon contributions are spurious. Within the original integrand reduction algorithm, their computation is needed because they appear in the subtractions at the integrand level required for the evaluation of lower-point contributions. A 5-point residue only has one coefficient, which can easily be computed by evaluating the numerator of the integrand on the corresponding 5-ple cut. Within the Laurent expansion approach, the subtraction terms coming from five-point residues always vanish in the asymptotic limits we consider, therefore their computation can be skipped. For this reason, in the library NinJa the computation of pentagons is disabled by default, even though it can be enabled for debugging purposes, as explained in Section 4.3.

4-point residues The coefficient $c_{0}$ of a box contribution $\Delta_{i_{1} i_{2} i_{3} i_{4}}$ can be determined via four-dimensional quadruple cuts [5]. A quadruple cut $D_{i_{1}}=\cdots=D_{i_{4}}=0$ in four dimensions (i.e. $\bar{q}=q, \mu^{2}=0$ ) has two solutions, $q_{+}$and $q_{-}$. The coefficient $c_{0}$ can be expressed in terms of these solutions as

$$
c_{0}=\frac{1}{2}\left(\left.\frac{\mathcal{N}(q)}{\prod_{j \neq i_{1}, i_{2}, i_{3}, i_{4}} D_{j}}\right|_{q=q_{+}}+\left.\frac{\mathcal{N}(q)}{\prod_{j \neq i_{1}, i_{2}, i_{3}, i_{4}} D_{j}}\right|_{q=q_{-}}\right) .
$$

Given the simplicity of Eq. (16), this is the only coefficient which NinJA computes in the same way as the traditional algorithm. The coefficient $c_{4}$ can instead be determined by evaluating the integrand on $d$-dimensional quadruple cuts in the asymptotic limit of large $\mu^{2}$ [35]. A $d$-dimensional quadruple cut has an infinite number of solutions which can be parametrized by the $(-2 \epsilon)$-dimensional variable $\mu^{2}$. These solutions become simpler in the considered limit, 
namely

$$
q_{ \pm}^{\nu}=-p_{i_{1}}^{\nu}+a^{\nu} \pm \sqrt{\mu^{2}+\beta} v_{\perp}^{\nu} \stackrel{\mu^{2} \rightarrow \infty}{=} \pm \sqrt{\mu^{2}} v_{\perp}^{\nu}+\mathcal{O}(1)
$$

where the vector $a^{\nu}$ and the constant $\beta$ are fixed by the cut conditions. The coefficient $c_{4}$ is non-vanishing only if the rank of the numerator is greater or equal to the number of loop denominators. In a renormalizable theory, it can be found in the $\mu^{2} \rightarrow \infty$ asymptotic limit as the leading term of the Laurent expansion of the integrand

$$
\left.\frac{\mathcal{N}\left(q, \mu^{2}\right)}{\prod_{j \neq i_{1}, i_{2}, i_{3}, i_{4}} D_{j}}\right|_{q=\sqrt{\mu^{2}} v_{\perp}+\mathcal{O}(1)}=c_{4} \mu^{4}+\mathcal{O}\left(\mu^{3}\right)
$$

The other coefficients of the boxes are spurious and, since they neither contribute to the final result nor to the subtraction terms, their computation can be skipped.

3-point residues The coefficients of the residues of a generic triangle contribution $\Delta_{i_{1} i_{2} i_{3}}$ can be determined by evaluating the integrand on the solutions of the corresponding $d$ dimensional triple cut [32]. These can be parametrized by the variable $\mu^{2}$ and the free parameter $t$,

$$
q_{+}^{\nu}=-p_{i_{1}}^{\nu}+a^{\nu}+t e_{3}^{\nu}+\frac{\beta+\mu^{2}}{2 t\left(e_{3} \cdot e_{4}\right)} e_{4}^{\nu}, \quad q_{-}=-p_{i_{1}}^{\nu}+a^{\nu}+\frac{\beta+\mu^{2}}{2 t\left(e_{3} \cdot e_{4}\right)} e_{3}^{\nu}+t e_{4}^{\nu},
$$

where the vector $a^{\nu}$ and the constant $\beta$ are fixed by the cut conditions $D_{i_{1}}=D_{i_{2}}=D_{i_{3}}=0$. The momentum $a^{\nu}$ is a linear combination of $e_{1}$ and $e_{2}$ and is therefore orthogonal to $e_{3}$ and $e_{4}$. On these solutions, the non-vanishing contributions to the integrand decomposition are the ones of the residue $\Delta_{i_{1} i_{2} i_{3}}$, as well as the ones of the boxes and pentagons which share the three cut denominators. However, after performing a Laurent expansion for large $t$ and dropping the terms which vanish in this limit, the pentagon contributions vanish, while the box contributions are constant in $t$ but they also vanish when taking the average between the parametrizations $q_{+}$and $q_{-}$of Eq. (19). More explicitly,

$$
\begin{aligned}
\frac{\mathcal{N}\left(q_{ \pm}, \mu^{2}\right)}{\prod_{j \neq i_{1}, i_{2}, i_{3}} D_{j}} & =\Delta_{i_{1} i_{2} i_{3}}+\sum_{j} \frac{\Delta_{i_{1} i_{2} i_{3} j}}{D_{j}}+\sum_{j k} \frac{\Delta_{i_{1} i_{2} i_{3} j k}}{D_{j} D_{k}} \\
& =\Delta_{i_{1} i_{2} i_{3}}+d_{1}^{ \pm}+d_{2}^{ \pm} \mu^{2}+\mathcal{O}(1 / t), \quad \text { with } d_{i}^{+}+d_{i}^{-}=0 .
\end{aligned}
$$

Moreover, the expansion of the integrand is given by

$$
\begin{aligned}
& \frac{\mathcal{N}\left(q_{+}, \mu^{2}\right)}{\prod_{j \neq i_{1}, i_{2}, i_{3}} D_{j}}=n_{0}^{+}+n_{7}^{+} \mu^{2}+\left(n_{4}+n_{9} \mu^{2}\right) t+n_{5} t^{2}+n_{6} t^{3}+\mathcal{O}(1 / t), \\
& \frac{\mathcal{N}\left(q_{-}, \mu^{2}\right)}{\prod_{j \neq i_{1}, i_{2}, i_{3}} D_{j}}=n_{0}^{-}+n_{7}^{-} \mu^{2}+\left(n_{1}+n_{8} \mu^{2}\right) t+n_{2} t^{2}+n_{3} t^{3}+\mathcal{O}(1 / t)
\end{aligned}
$$

and it has the same polynomial behavior as the expansion of the residue $\Delta_{i_{1} i_{2} i_{3}}$,

$$
\begin{aligned}
& \Delta_{i_{1} i_{2} i_{3}}\left(q_{+}, \mu^{2}\right)=c_{0}+c_{7} \mu^{2}+\left(c_{4}+c_{9} \mu^{2}\right)\left(e_{3} \cdot e_{4}\right) t+c_{5}\left(e_{3} \cdot e_{4}\right)^{2} t^{2}+c_{6}\left(e_{3} \cdot e_{4}\right)^{3} t^{3}+\mathcal{O}(1 / t), \\
& \Delta_{i_{1} i_{2} i_{3}}\left(q_{-}, \mu^{2}\right)=c_{0}+c_{7} \mu^{2}+\left(c_{1}+c_{8} \mu^{2}\right)\left(e_{3} \cdot e_{4}\right) t+c_{2}\left(e_{3} \cdot e_{4}\right)^{2} t^{2}+c_{3}\left(e_{3} \cdot e_{4}\right)^{3} t^{3}+\mathcal{O}(1 / t) .
\end{aligned}
$$


By comparison of Eq. (20), (21) and (22) one can directly identify the ten triangle coefficients as the corresponding terms of the expansion of the integrand,

$$
c_{0,7}=\frac{1}{2}\left(n_{0,7}^{+}+n_{0,7}^{-}\right), \quad c_{1,4,8,9}=\frac{n_{1,4,8,9}}{\left(e_{3} \cdot e_{4}\right)}, \quad c_{2,5}=\frac{n_{2,5}}{\left(e_{3} \cdot e_{4}\right)^{2}}, \quad c_{3,6}=\frac{n_{3,6}}{\left(e_{3} \cdot e_{4}\right)^{3}} .
$$

Hence, with the Laurent expansion method, the determination of the 3-point residues does not require any subtraction of higher-point terms.

2-point residues The coefficients of a generic 2-point residue $\Delta_{i_{1} i_{2}}$ can be evaluated on the on-shell solutions of the corresponding double cut $D_{i_{1}}=D_{i_{2}}=0$, which can be parametrized as

$$
\begin{aligned}
& q_{+}=-p_{0}+x e_{1}^{\nu}+\left(\alpha_{0}+x \alpha_{1}\right) e_{2}^{\nu}+t e_{3}^{\nu}+\frac{\beta_{0}+\beta_{1} x+\beta_{2} x^{2}+\mu^{2}}{2 t\left(e_{3} \cdot e_{4}\right)} e_{4}^{\nu}, \\
& q_{-}=-p_{0}+x e_{1}^{\nu}+\left(\alpha_{0}+x \alpha_{1}\right) e_{2}^{\nu}+\frac{\beta_{0}+\beta_{1} x+\beta_{2} x^{2}+\mu^{2}}{2 t\left(e_{3} \cdot e_{4}\right)} e_{3}^{\nu}+t e_{4}^{\nu},
\end{aligned}
$$

in terms of the three free parameters $x, t$ and $\mu^{2}$, while the constants $\alpha_{i}$ and $\beta_{i}$ are fixed by the on-shell conditions. After evaluating the integrand on these solutions and performing a Laurent expansion for $t \rightarrow \infty$, the only non-vanishing subtraction terms come from the triangles,

$$
\begin{aligned}
\frac{\mathcal{N}\left(q_{ \pm}, \mu^{2}\right)}{\prod_{j \neq i_{1}, i_{2}} D_{j}} & =\Delta_{i_{1} i_{2}}+\sum_{j} \frac{\Delta_{i_{1} i_{2} j}}{D_{j}}+\sum_{j k} \frac{\Delta_{i_{1} i_{2} j k}}{D_{j} D_{k}}+\sum_{j k l} \frac{\Delta_{i_{1} i_{2} j k l}}{D_{j} D_{k} D_{l}} \\
& =\Delta_{i_{1} i_{2}}+\sum_{j} \frac{\Delta_{i_{1} i_{2} j}}{D_{j}}+\mathcal{O}(1 / t) .
\end{aligned}
$$

Even though the integrand and the subtraction terms are rational functions, in the asymptotic limit they both have the same polynomial behavior as the residue, namely

$$
\begin{aligned}
\frac{\mathcal{N}\left(q_{+}, \mu^{2}\right)}{\prod_{j \neq i_{1}, i_{2}} D_{j}}= & n_{0}+n_{9} \mu^{2}+n_{1} x+n_{2} x^{2}-\left(n_{5}+n_{8} x\right) t+n_{6} t^{2}+\mathcal{O}(1 / t) \\
\frac{\mathcal{N}\left(q_{-}, \mu^{2}\right)}{\prod_{j \neq i_{1}, i_{2}} D_{j}}= & n_{0}+n_{9} \mu^{2}+n_{1} x+n_{2} x^{2}-\left(n_{3}+n_{7} x\right) t+n_{4} t^{2}+\mathcal{O}(1 / t) \\
\frac{\Delta_{i_{1} i_{2} j}\left(q_{+}, \mu^{2}\right)}{D_{j}}= & c_{s_{3}, 0}^{(j)}+c_{s_{3}, 9}^{(j)} \mu^{2}+c_{s_{3}, 1}^{(j)} x+c_{s_{3}, 2}^{(j)} x^{2}-\left(c_{s_{3}, 5}^{(j)}+c_{s_{3}, 8}^{(j)} x\right) t+c_{s_{3}, 6}^{(j)} t^{2}+\mathcal{O}(1 / t) \\
\frac{\Delta_{i_{1} i_{2} j}\left(q_{-}, \mu^{2}\right)}{D_{j}}= & c_{s_{3}, 0}^{(j)}+c_{s_{3}, 9}^{(j)} \mu^{2}+c_{s_{3}, 1}^{(j)} x+c_{s_{3}, 2}^{(j)} x^{2}-\left(c_{s_{3}, 3}^{(j)}+c_{s_{3}, 7}^{(j)} x\right) t+c_{s_{3}, 4}^{(j)} t^{2}+\mathcal{O}(1 / t) \\
\Delta_{i_{1} i_{2}}\left(q_{+}, \mu^{2}\right)= & c_{0}+c_{9} \mu^{2}+c_{1}\left(e_{1} \cdot e_{2}\right) x+c_{2}\left(e_{1} \cdot e_{2}\right)^{2} x^{2} \\
& +\left(c_{5}+c_{8}\left(e_{1} \cdot e_{2}\right) x\right)\left(e_{3} \cdot e_{4}\right) t+c_{6}\left(e_{3} \cdot e_{4}\right)^{2} t^{2}+\mathcal{O}(1 / t) \\
\Delta_{i_{1} i_{2}}\left(q_{-}, \mu^{2}\right)= & c_{0}+c_{9} \mu^{2}+c_{1}\left(e_{1} \cdot e_{2}\right) x+c_{2}\left(e_{1} \cdot e_{2}\right)^{2} x^{2} \\
& +\left(c_{3}+c_{7}\left(e_{1} \cdot e_{2}\right) x\right)\left(e_{3} \cdot e_{4}\right) t+c_{4}\left(e_{3} \cdot e_{4}\right)^{2} t^{2}+\mathcal{O}(1 / t)
\end{aligned}
$$


The coefficients $c_{s_{3}, i}^{(j)}$ of the expansion of the subtractions terms in Eq.s (27) are known parametric functions of the triangle coefficients. Hence, the subtraction of the triangle contributions can be implemented by applying coefficient-level corrections to the terms appearing in the expansion of the integrand. More explicitly, by inserting Eq.s (26), (27) and (28) in Eq. (25) one gets

$$
\begin{aligned}
c_{0,9} & =n_{0,9}-\sum_{j} c_{s_{3}, 0,9}^{(j)}, \\
c_{1,3,5} & =\frac{1}{\left(e_{1} \cdot e_{2}\right)}\left(n_{1,3,5}-\sum_{j} c_{s_{3}, 1,3,5}^{(j)}\right), \\
c_{2,4,6,7,8} & =\frac{1}{\left(e_{1} \cdot e_{2}\right)^{2}}\left(n_{2,4,6,7,8}-\sum_{j} c_{s_{3}, 2,4,6,7,8}^{(j)}\right) .
\end{aligned}
$$

1-point residues The only non-spurious coefficient $c_{0}$ of a tadpole residue $\Delta_{i_{1}}$ can be computed by evaluating the integrand on solutions of the single cut $D_{i_{1}}=0$. For this purpose, one can consider 4-dimensional solutions of the form

$$
q_{+}^{\nu}=-p_{i_{1}}^{\nu}+t e_{3}^{\nu}+\frac{m_{i_{1}}^{2}}{2 t\left(e_{3} \cdot e_{4}\right)} e_{4}^{\nu},
$$

parametrized by the free variable $t$. In the asymptotic limit $t \rightarrow \infty$, only bubble and triangle subtraction terms are non-vanishing,

$$
\begin{aligned}
\frac{\mathcal{N}\left(q_{+}\right)}{\prod_{j \neq i_{1}} D_{j}} & =\Delta_{i_{1}}+\sum_{j} \frac{\Delta_{i_{1} j}}{D_{j}}+\sum_{j k} \frac{\Delta_{i_{1} j k}}{D_{j} D_{k}}+\sum_{j k l} \frac{\Delta_{i_{1} j k l}}{D_{j} D_{k} D_{l}} \\
& =\Delta_{i_{1}}+\sum_{j} \frac{\Delta_{i_{1} j}}{D_{j}}+\sum_{j k} \frac{\Delta_{i_{1} j k}}{D_{j} D_{k}}+\mathcal{O}(1 / t) .
\end{aligned}
$$

Similarly to the case of the 2-point residues, in this limit the integrand and the subtraction terms exhibit the same polynomial behavior as the residue, i.e.

$$
\begin{aligned}
\frac{\mathcal{N}\left(q_{+}\right)}{\prod_{j \neq i_{1}} D_{j}} & =n_{0}+n_{4}\left(e_{3} \cdot e_{4}\right) t+\mathcal{O}(1 / t) \\
\frac{\Delta_{i_{1} j}\left(q_{+}\right)}{D_{j}} & =c_{s_{2}, 0}^{(j)}+c_{s_{2}, 4}^{(j)}\left(e_{3} \cdot e_{4}\right) t+\mathcal{O}(1 / t) \\
\frac{\Delta_{i_{1} j k}\left(q_{+}\right)}{D_{j} D_{k}} & =c_{s_{3}, 0}^{(j k)}+c_{s_{3}, 4}^{(j k)}\left(e_{3} \cdot e_{4}\right) t+\mathcal{O}(1 / t) \\
\Delta_{i_{1}}\left(q_{+}\right) & =c_{0}^{\left(i_{1}\right)}+c_{4}^{\left(i_{1}\right)} t+\mathcal{O}(1 / t) .
\end{aligned}
$$

Putting everything together, the coefficient of the tadpole integral can be identified with the corresponding one in the expansion of the integrand, corrected by coefficient-level subtractions from bubbles and triangles

$$
c_{0}=n_{0}-\sum_{j} c_{s_{2}, 0}^{(j)}-\sum_{j k} c_{s_{3}, 0}^{(j k)}
$$

The subtraction terms $c_{s_{2}, 0}^{(j)}$ and $c_{s_{3}, 0}^{(j k)}$, coming from 2-point and 3-point contributions respectively, are known parametric functions of the coefficients of the corresponding higher-point residues. 


\section{Semi-numerical implementation}

The $\mathrm{C}++$ library NinJA provides a semi-numerical implementation of the Laurent expansion method described in Section 2. The Laurent series expansion is typically an analytic operation, but since a one-loop integrand is a rational function of the loop variables, its expansion can be obtained via a partial fraction decomposition between the numerator and the denominators. This is implemented in NINJA via a simplified polynomial-division algorithm, which takes as input the coefficients of a parametric expansion of the numerator $\mathcal{N}$ and computes the leading terms of the quotient of the polynomial division with respect to the uncut denominators. In this section we describe the input needed for the reduction performed by NiNJA and we give further details about the implementation of the reduction.

All the types, classes and functions provided by the NinJA library are defined inside the ninja namespace. In particular, the types Real and Complex are aliases for double and std: : complex<double>, unless the library was compiled in quadruple precision. Classes for real and complex momenta are defined as RealMomentum and ComplexMomentum respectively. They are wrappers of four-dimensional arrays of the corresponding floating-point types, which overload arithmetic and subscript operators. More in detail, an instance $\mathrm{p}$ of one of these classes represents a momentum $p$ according to the representation

$$
\mathrm{p}=\{\mathrm{p}[0], \mathrm{p}[1], \mathrm{p}[2], \mathrm{p}[3]\}=\left\{E_{p}, x_{p}, y_{p}, z_{p}\right\},
$$

i.e. with the energy in the zeroth component, followed by the spatial components.

\section{$3.1 \quad$ Input}

The inputs needed from the reduction algorithm implemented in NINJA are the momenta $p_{i}$ and the masses $m_{i}$ of the loop denominators defined in Eq. (2), besides the numerator $\mathcal{N}\left(q, \mu^{2}\right)$ of the integrand. The latter must be cast in four different forms, one of which is optional. The $\mathrm{C}++$ implementation requires the numerator to be an instance of a class inherited from the abstract class ninja: : Numerator. The latter defined as

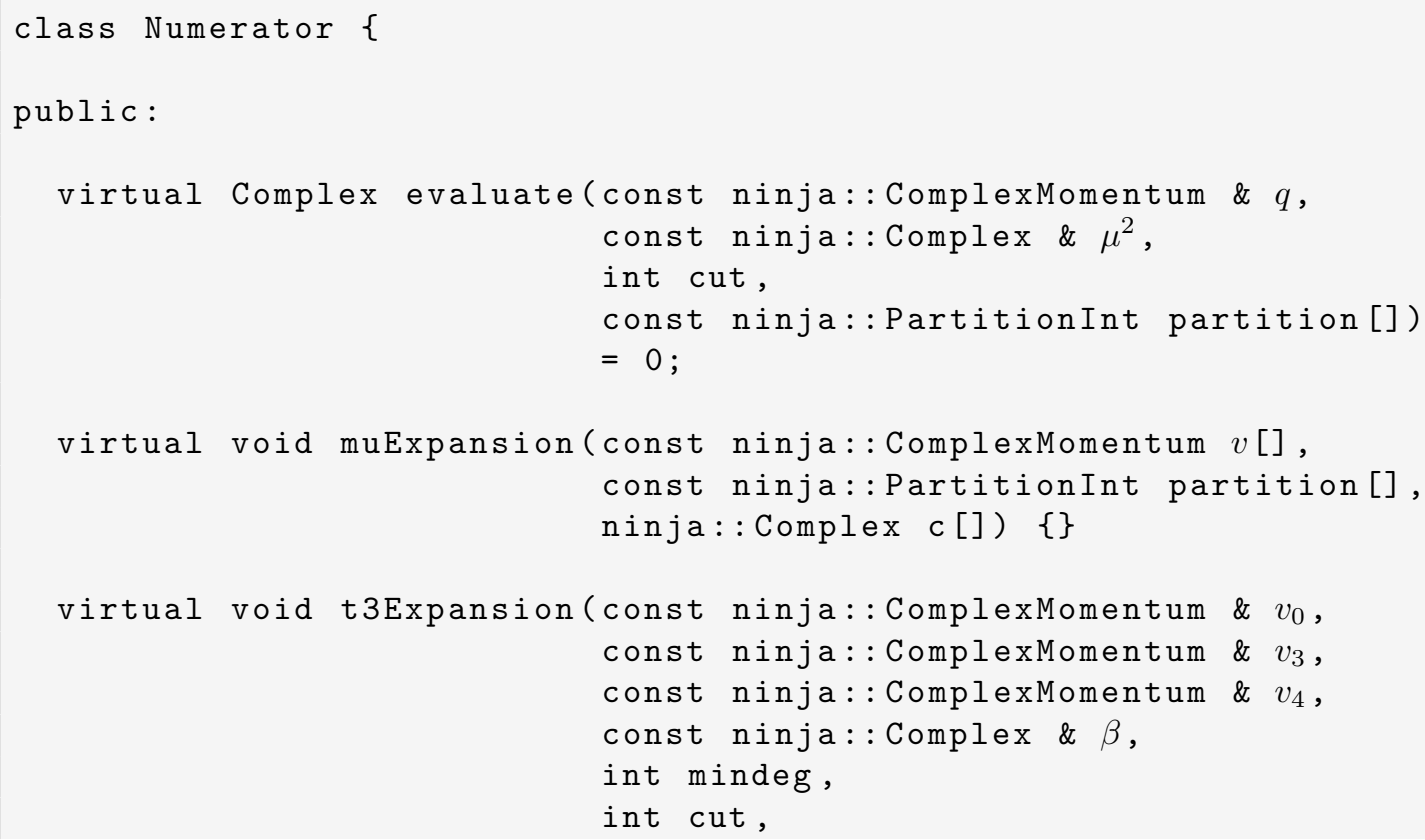




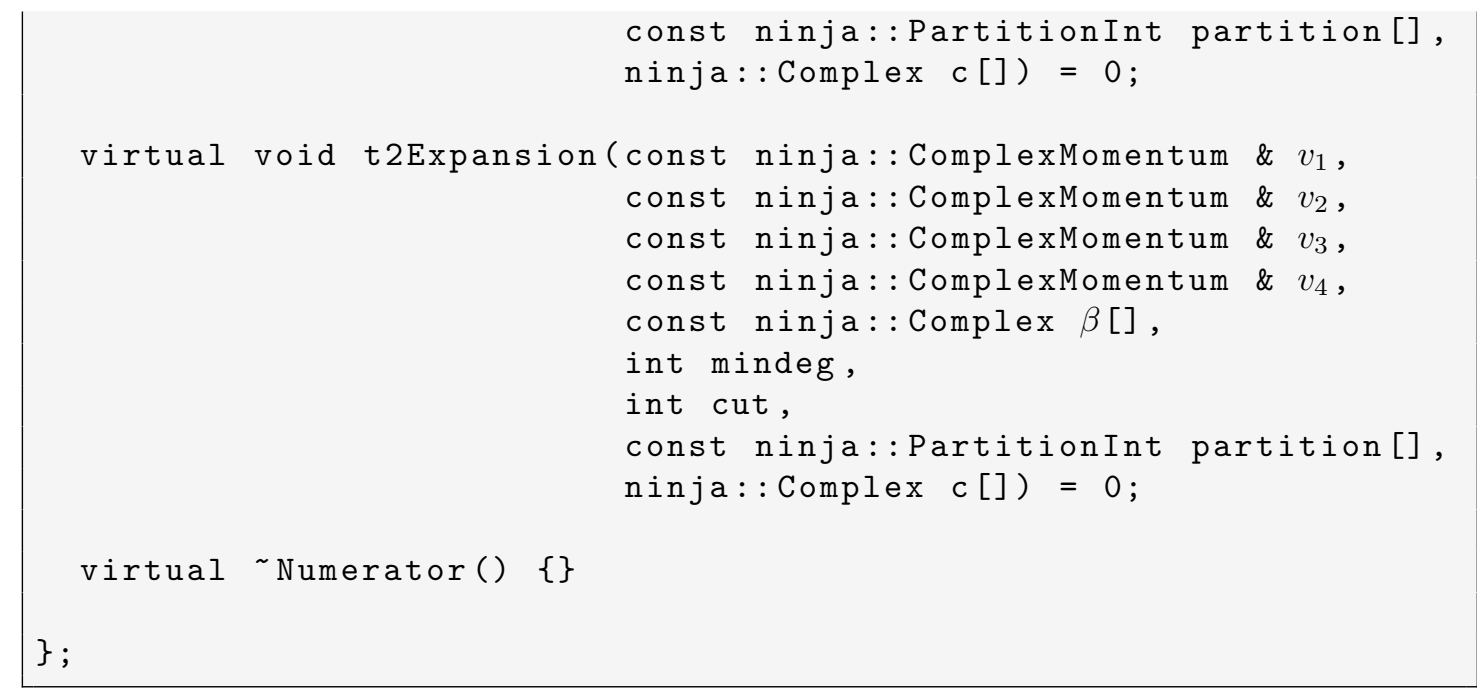

The input parameters cut and partition are common to more methods and give information about the multiple cut where NiNJA is currently evaluating the numerator. Although this information is not always necessary, there might be occasions where it could be useful for an efficient evaluation of the numerator. The integer cut is equal to $k$ if the numerator is being evaluated on a $k$-ple cut, with $k=1,2,3,4$. This parameter is not given in the method muExpansion because the latter is always evaluated on quadruple cuts (i.e. cut $=4$ ). The parameter partition points to an array of integers (namely of integer type ninja: :PartitionInt), with length equal to cut, containing the indexes of the cut numerators. If the user asks to perform a global test (see Section 4.3), the numerator will also be evaluated outside the solutions of the multiple cuts, in which case the parameter cut will be set to zero. As an example, if the method t3Expansion is evaluated on the 3-ple cut $D_{0}=D_{2}=D_{5}=0$ for the determination of the 3 -point residue $\Delta_{025}$, we will have cut $=3$, partition[0] $=0$, partition[1] $=2$, and partition [2] $=5$. The returned expansion only needs to be valid for the cut specified (e.g. $D_{0}=D_{2}=D_{5}=0$ in the previous example).

Here is a detailed description of each method of the class ninja: :Numerator, for a generic numerator of an $n$-point integrand of rank $r$. If the analytic expression of the integrand is available, all these methods can be easily generated with the help of the simple PYTHON package NinJANumGen, which is distributed with the library and whose usage is described in Section 4.2 and in Appendix A. The details which follow are only needed to those who prefer to provide an alternative implementation of the required methods without NinJANUMGEN.

\section{The method Numerator: : evaluate $\left(q, \mu^{2}\right.$, cut, partition)}

It must return the value of the numerator $\mathcal{N}\left(q, \mu^{2}\right)$ evaluated at the (complex) values of $q$ and $\mu^{2}$ given as input.

\section{The method Numerator: :muExpansion ( $v$, partition, c)}

This is used for the computation of the Laurent expansion in $\mu^{2}$ required to obtain the coefficient $c_{4}$ of the boxes. In the renormalizable case, this method should compute the leading term of a parametric expansion in $t$ of the integrand defined by

$$
q^{\nu} \rightarrow t v_{\perp}^{\nu}, \quad \mu^{2} \rightarrow t^{2} v_{\perp}^{2}
$$


where $v_{\perp}$ is given by $v$ [0], i.e. by the zeroth entry of the array of momenta $v$. For renormalizable theories this array will therefore only contain at most one element. The method should write the leading coefficient of the expansion in the zeroth entry of the array pointed by the parameter c, i.e.

$$
c[0]=\mathcal{N}\left[t^{r}\right]
$$

The generalization of this method to the higher-rank case is described in Appendix B. The implementation of this method is only required when $r \geq n$. It is also not needed if the user chooses to disable the $\mu^{2}$-expansion method for the boxes, but in that case more evaluations of the numerator will be needed and the computation of the pentagons will not be skipped.

The method Numerator: : t3Expansion $\left(v_{0}, v_{3}, v_{4}, \beta\right.$, mindeg, partition, c)

This method is used for the computation of the coefficients of the residues of both the triangles and the tadpoles. It must compute the coefficients of the terms $t^{j} \mu^{2 k}$ for $j \in\{r, r-1, r-$ $2, \ldots, r-$ mindeg $\}$, given by substituting into the numerator the parametric expansion of the loop momentum defined by

$$
q^{\nu} \rightarrow v_{0}^{\nu}+t v_{3}^{\nu}+\frac{\beta+\mu^{2}}{t} v_{4}^{\nu}, \quad v_{3}^{2}=v_{4}^{2}=0, \quad\left(v_{3} \cdot v_{4}\right)=\frac{1}{2},
$$

as a function of the momenta $v_{i}^{\nu}$ and the constant $\beta$ which are passed as parameters. The maximum value of the parameter mindeg is $r-n+3$. Since in a renormalizable theory $r \leq n$, and by definition of rank we have $j+2 k \leq r$, in this case at most 6 terms can be non-vanishing in the specified range of $j$. The method should write these terms in the entries of the array pointed by $\mathrm{c}$, ordered by decreasing powers of $t$. Terms with the same power of $t$ should be ordered by increasing powers of $\mu^{2}$. A pseudo-implementation will therefore look like

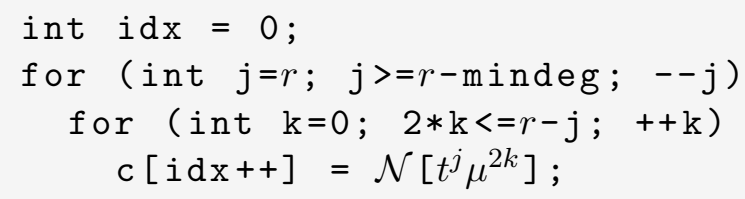

The method Numerator: : t2Expansion $\left(v_{1}, v_{2}, v_{3}, v_{4}, \beta\right.$, mindeg, partition, c)

In the current version of NINJA, this method is called during the computation of the coefficients of the bubbles. It must compute the coefficients of the terms $t^{j} x^{l} \mu^{2 k}$ for $j \in$ $\{r, r-1, \ldots, r-$ mindeg $\}$, given by the expansion

$$
\begin{aligned}
& q^{\nu} \rightarrow v_{1}^{\nu}+x v_{2}^{\nu}+t v_{3}^{\nu}+\frac{\beta_{0}+\beta_{1} x+\beta_{2} x^{2}+\mu^{2}}{t} v_{4}^{\nu}, \\
& v_{2} \cdot v_{3}=v_{2} \cdot v_{4}=v_{3}^{2}=v_{4}^{2}=0, \quad\left(v_{3} \cdot v_{4}\right)=\frac{1}{2}
\end{aligned}
$$

as a function of the momenta $v_{i}^{\nu}$ and the constants $\beta_{i} \equiv \beta$ [i], which are passed as parameters to the method. The maximum value of mindeg is $r-n+2$. In a renormalizable theory, this implies that one can have at most 7 non-vanishing terms in this range of $j$. It is worth observing that the expansion in Eq. (39) can be obtained from the previous one in Eq. (38) with the substitutions

$$
v_{0}^{\nu} \rightarrow v_{1}^{\nu}+x v_{2}^{\nu}, \quad \beta \rightarrow \beta_{0}+\beta_{1} x+\beta_{2} x^{2}, \quad v_{2} \cdot v_{3}=v_{2} \cdot v_{4}=0 .
$$


The terms of the expansion must be stored in the entries of the array pointed by c, ordered by decreasing powers of $t$. Terms with the same power of $t$ should be ordered from the lowest to the highest with respect to the lexicographical order in the variables $\left(x, \mu^{2}\right)$. A pseudo-implementation will have the form

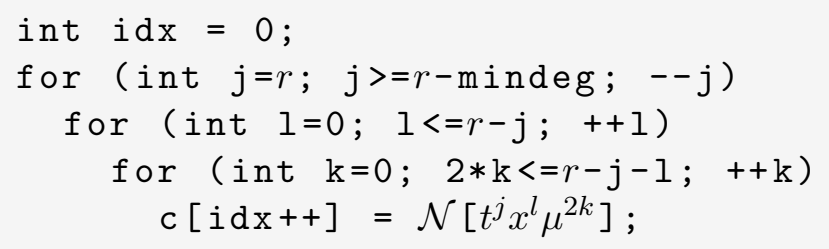

\subsection{Reduction via polynomial division}

For every phase-space point, NinJA at run-time computes the parametric solutions of the multiple cuts corresponding to each residue. The Laurent expansion of the integrand on these solutions is performed via a simplified polynomial division between the expansion of the numerator and the set of the uncut denominators. The coefficients of this expansion are corrected by the coefficient-level subtractions appearing in Eq. (29) and (36). The nonspurious coefficients are finally multiplied by the corresponding Master Integrals in order to obtain the integrated result as in Eq. (15).

The coefficients of the expansions of the numerator are written on a contiguous array by the numerator methods described in Section 3.1. The Laurent expansion is obtained via a simplified polynomial division. The latter is performed in-place on the same array, keeping only the elements which are needed for the final result. A possible implementation for an univariate expansion, with a numerator

$$
\mathcal{N}=\operatorname{num}[0] t^{r}+\operatorname{num}[1] t^{r-1}+\ldots+\text { num [nterms-1] } t^{r-\text { nterms }+1}+\mathcal{O}\left(t^{r-\text { nterms }}\right)
$$

and denominator

$$
D=\mathrm{d}[0] t+\mathrm{d}[1]+\mathrm{d}[2] \frac{1}{t}
$$

would have the form

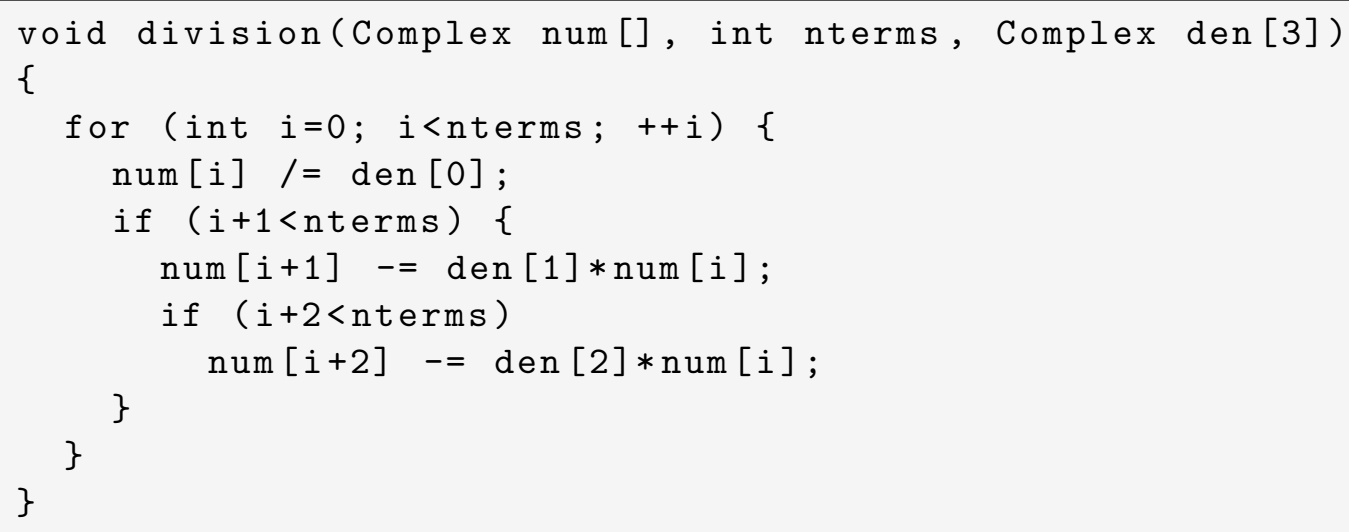

One can check that this routine correctly replaces the first nterms elements of the array num with the first nterms leading elements of the Laurent expansion of $\mathcal{N} / D$. The actual implementation in NinJA, having to deal with multivariate expansions, is significantly more 
involved than the division procedure presented here. Nevertheless, it qualitatively follows the same algorithm.

The coefficients obtained by the division are then corrected by the coefficient-level subtractions and thus identified with the corresponding coefficients of the residues, as explained in Section 2. Once the reduction is complete, the non-spurious coefficients are multiplied by the corresponding Master Integrals.

\subsection{Master Integrals}

NinJA calls the routines implementing the Master Integrals through a generic interface which, as in the case of the numerator, is defined in the $\mathrm{C}++$ code via an abstract class (called IntegralLibrary). This allows one to use any integral library which can be specified at run-time. More details on the implementation of this interface are given in Appendix C. The current version of NINJA already implements it for two integral libraries.

The first built-in interface, ninja: : AvHOneLoop, is a $\mathrm{C}++$ wrapper of the routines of the OneLoop library $[16,25]$. This wrapper caches every computed integral allowing constanttime lookup of their values from their arguments. The caching of the integrals can significantly speed up the computation, especially for complex processes. Every instance of the class AvHOneLoop has an independent cache of Master Integrals (hence, one may safely use it in multi-threaded applications by using one instance of the class per thread).

The second implementation of the interface, ninja: :LoopTools, uses instead the LoopTools library [15], which already has an internal cache of computed integrals.

\section{Basic usage}

In this section we explain how to use the library for the computation of a generic one-loop integral. NinJA can be interfaced to any one-loop generator capable of providing the input it needs, and in particular to packages which can reconstruct the analytic dependence of the numerators on the loop momentum. An interface for the one-loop package GoSam [20] is already built in the library, and has been used for highly non-trivial phenomenological computations [26, 27]. An interface with the package FormCALC [15] is currently under development. The author is open to give his assistance in interfacing other packages as well.

In this paper we focus on the usage of NinJA as a standalone library. We will show how to generate the numerator methods needed as input, starting from an analytic expression of the numerator, with the help of the PYTHON package NinJANuMGEN which is distributed with the library. We will then explain how to perform the reduction, and how to set the main available options.

\subsection{Installation}

NINJA can be obtained at the url http://ninja.hepforge.org. The library is distributed with its source code using the GNU build system (also known as AUTOTOOLS). It can be compiled and installed with the shell commands

. / configure

make

make install 
This will typically install the library and the header files in sub-directories of /usr/local. The --prefix option can be used in order to specify a different installation path. In this case, you might need to update your LD_LIBRARY_PATH (or DYLD_LIBRARY_PATH on MAC OS) environment variable accordingly. In order to use NINJA for the production of phenomenological results, one must interface it with a library of Master Integrals. As already mentioned, interfaces to the ONELOOP and LOOPTOOLS libraries are provided (see Appendix C for interfacing a different library). They can be enabled by passing to the configure script the options --with-avholo[=FLAGS] and --with-looptools [=FLAGS]. For instance, the following commands

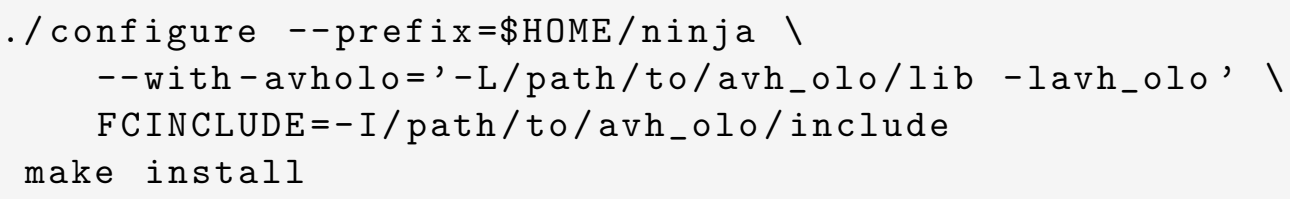

will install all the files in sub-directories of \$HOME/ninja and build the interface with the ONELOOP library, which must be already installed and linkable with the flags specified with the --with-avholo option. We also specified the FCINCLUDE variable with the flags which are needed to find ForTRAN-90 modules when they are not installed in a default directory.

Given the importance of numerical precision in the calculation of scattering amplitudes, there is also the option to compile the library in quadruple precision (--with-quadruple), which uses the GCC LIBQUADMATH library. By using the ninja: : Real and ninja: :Complex floating point types, the same source code can be compiled both in double and quadruple precision, depending on how NinJA has been configured.

A full list of optional arguments for the configure script can be obtained with the command ./configure --help. While most of them are common to every package distributed with the GNU build system, some are instead specific to the NinJA library and they are described in Table 1. In most of the cases, only the options for interfacing the integral libraries should be needed.

The user can also optionally install the PYTHON package NinjaNumGen, which allows one to easily generate the input needed by NinJA. The package can be used both as a script and as a PYTHON module, and it could also be useful for interfacing NinJA to existing one-loop packages. In order to install the package, move in the utils folder and type

python setup.py install

where, as usual, an installation prefix can be specified using --prefix. In this case one might need to update the PATH and PYTHONPATH environment variables accordingly. The package needs Form-4 [29,30], which should be installed separately by the user, in order to compute the expansions which are needed and for the generation of the corresponding source code.

Further information about the installation procedure can be found in the README file of the distribution.

\subsection{Writing the Integrand}

The most important input that the NinJA library needs, for the computation of a one-loop integral, is the numerator of the corresponding integrand, cast in a suitable form. This form has been described in detail in Section 3.1 but, as already mentioned, it can also be generated with the help of the package NinJANUMGEN. In this section we describe its usage as a script, 


\begin{tabular}{|c|c|}
\hline Option & Description \\
\hline --with-avholo[=FLAGS] & $\begin{array}{l}\text { Include an interface with the ONELOOP integral library [16, } \\
25] \text {, specifying the corresponding flags for dynamic linking. } \\
\text { If the FoRTRAN module avh_olo is not in a standard path, } \\
\text { one should add its directory to the FCINCLUDE variable when } \\
\text { using this option. }\end{array}$ \\
\hline --with-looptools [=FLAGS] & $\begin{array}{l}\text { Include an interface with the LoOPToOLS library [15] } \\
\text { (needs LoOPToOLS version } 2.9 \text { or higher), specifying the } \\
\text { coresponding flags for static linking. If the header file } \\
\text { clooptools.h is not in a standard path, one should add its } \\
\text { directory to the CPPFLAGS variable when using this option. }\end{array}$ \\
\hline --with-quadruple [=FLAGS] & $\begin{array}{l}\text { Compile the library in quadruple precision. This requires the } \\
\text { GCC LIBQUADMATH library and one can specify the corre- } \\
\text { sponding flags for the linker (-lquadmath by default). With } \\
\text { this option, the types ninja: : Real and ninja: : Complex } \\
\text { will be quadruple precision floating point numbers, and in- } \\
\text { cluding any public header file of the library will define the } \\
\text { macro NINJA_QUADRUPLE to } 1 \text { (it will not be defined other- } \\
\text { wise). The user should also make sure, when using this op- } \\
\text { tion, that the libraries of Master Integrals used by NiNJA are } \\
\text { compiled in quadruple precision, with floating point types } \\
\text { compatible with the ones of GCC. }\end{array}$ \\
\hline --enable-higher_rank & $\begin{array}{l}\text { Enable support for higher-rank numerators. This is not } \\
\text { needed for renormalizable theories. }\end{array}$ \\
\hline --disable-gosam & Do not include GoSAM interface. \\
\hline --disable-avholo_cache & $\begin{array}{l}\text { Do not include a cache of Master Integrals in the interface } \\
\text { with the ONELOOP library. }\end{array}$ \\
\hline
\end{tabular}

Table 1: Options and environment flags for the configure script. Only the options which modify the default behavior of NINJA are listed.

with a simple example. A more detailed list of options, allowing to fine-tune the output according to the user's needs, as well as the usage of the package as a PYTHON module, are described in Appendix A.

Let us define, as an example, the following 4-point one-loop integrand, with kinematics $k_{0}, k_{1} \rightarrow k_{2}, k_{3}$

$$
\begin{aligned}
\mathcal{I} & =\frac{\mathcal{N}\left(q, \mu^{2}\right)}{D_{0} D_{1} D_{2} D_{3}}=\frac{\left(q \cdot v_{1}\right)\left(q \cdot v_{2}\right)\left(q \cdot v_{3}\right)\left(q \cdot v_{4}\right)+\mu^{4}}{D_{0} D_{1} D_{2} D_{3}} \\
D_{0} & =\bar{q}^{2}-m_{0}^{2} \\
D_{1} & =\left(\bar{q}+k_{0}\right)^{2}-m_{1}^{2} \\
D_{2} & =\left(\bar{q}+k_{0}+k_{1}\right)^{2}-m_{2}^{2} \\
D_{3} & =\left(\bar{q}+k_{3}\right)^{2}-m_{3}^{2} .
\end{aligned}
$$

where $v_{i}$ are arbitrary reference vectors. In order to generate the methods declared in the ninja: : Numerator class, we first create a file mynum.frm containing a FoRM [28] expression 
for the numerator

* mynum.frm

$\mathrm{V} \mathrm{v} 1, \mathrm{v} 2, \mathrm{v} 3, \mathrm{v} 4$;

$\mathrm{V} Q$;

S Mu2;

L Diagram $=(\mathrm{Q} \cdot \mathrm{v} 1) *(\mathrm{Q} \cdot \mathrm{v} 2) *(\mathrm{Q} \cdot \mathrm{v} 3) *(\mathrm{Q} \cdot \mathrm{v} 4)+\mathrm{Mu} 2{ }^{\wedge} 2$;

and we run the script with the command

ninjanumgen mynum.frm --nlegs 4 --rank 4 -o mynum.cc

which creates the source file mynum.cc with the definition of the methods, optimized for fast numerical evaluation using the recent features of Form-4. The command also creates an header file mynum.hh, unless already present, which must contain the declaration of the numerator class. The latter will have the following form (where for brevity we replaced the parameters of each method with ellipses),

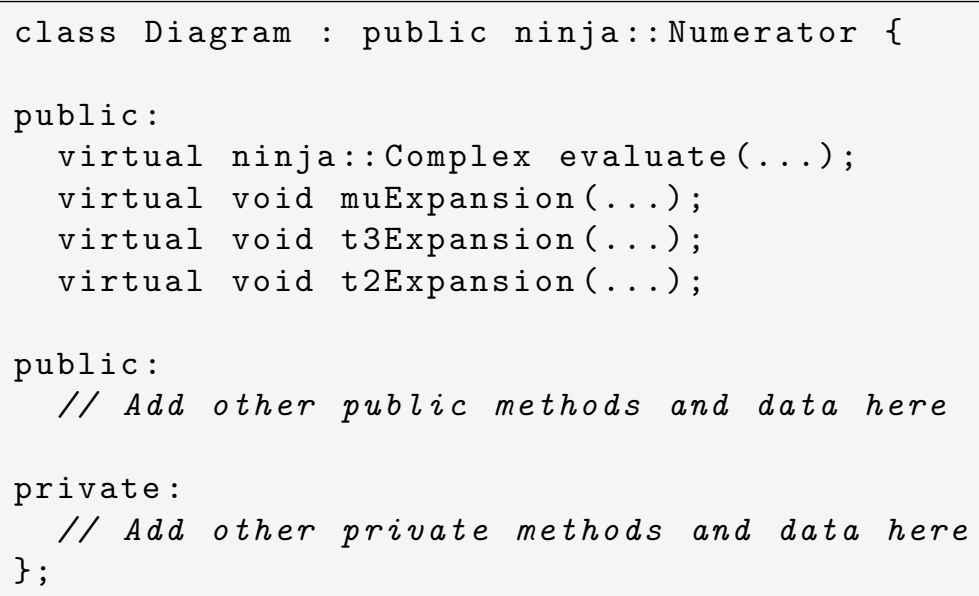

If the numerator expression depends on other momenta or parameters, these should be visible inside the definitions of the methods. In our example, the numerator depends on the reference vectors $v_{i}$ which appear in its Form expression. One possibility would be declaring these vectors as global variables, but a better alternative could be defining them as data members of the numerator class. In this example we will declare them as public data members by inserting the following code inside the class definition

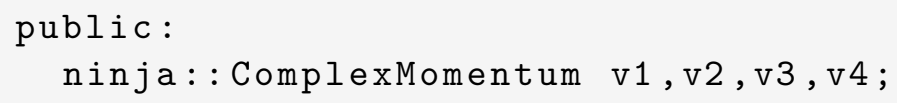

which defines the vectors as complex Lorentz momenta. This completes the generation of the input, which will allow NiNJA to compute the integral.

\subsection{Running the reduction}

In this subsection we describe the usage of NINJA for the reduction of a generated integrand, such as the one in the example of the previous subsection. With the help of simple examples, we show how to specify the input and how to control the run-time behavior of the procedures 
of the library. All the public header files of the library are installed in the sub-directory ninja of the include path in the installation directory.

\subsubsection{A simple example}

Here we show the contents of a file simple_test.cc which illustrate the basic usage of NiNJA for computing the integral defined in Eq. (40).

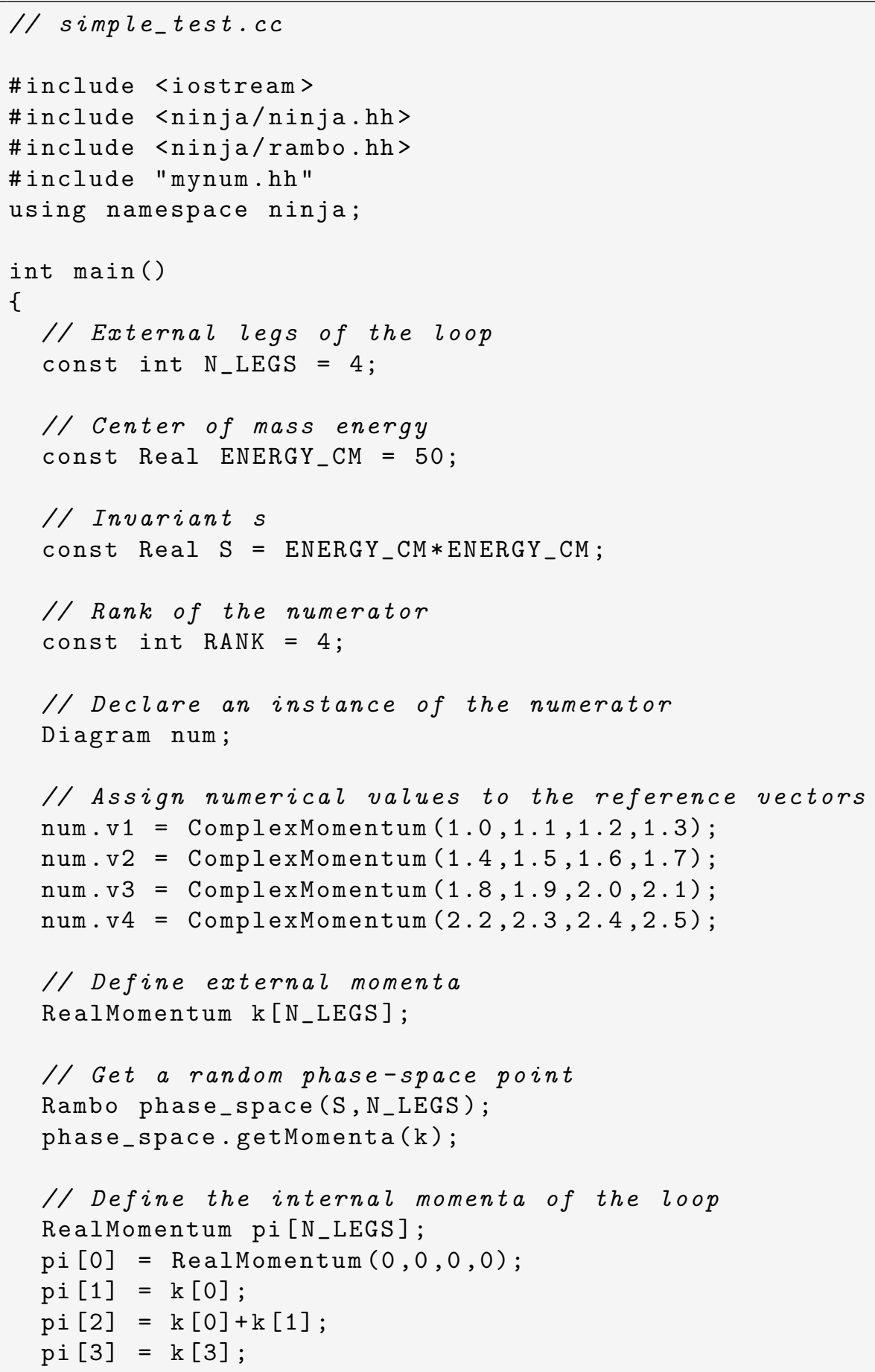




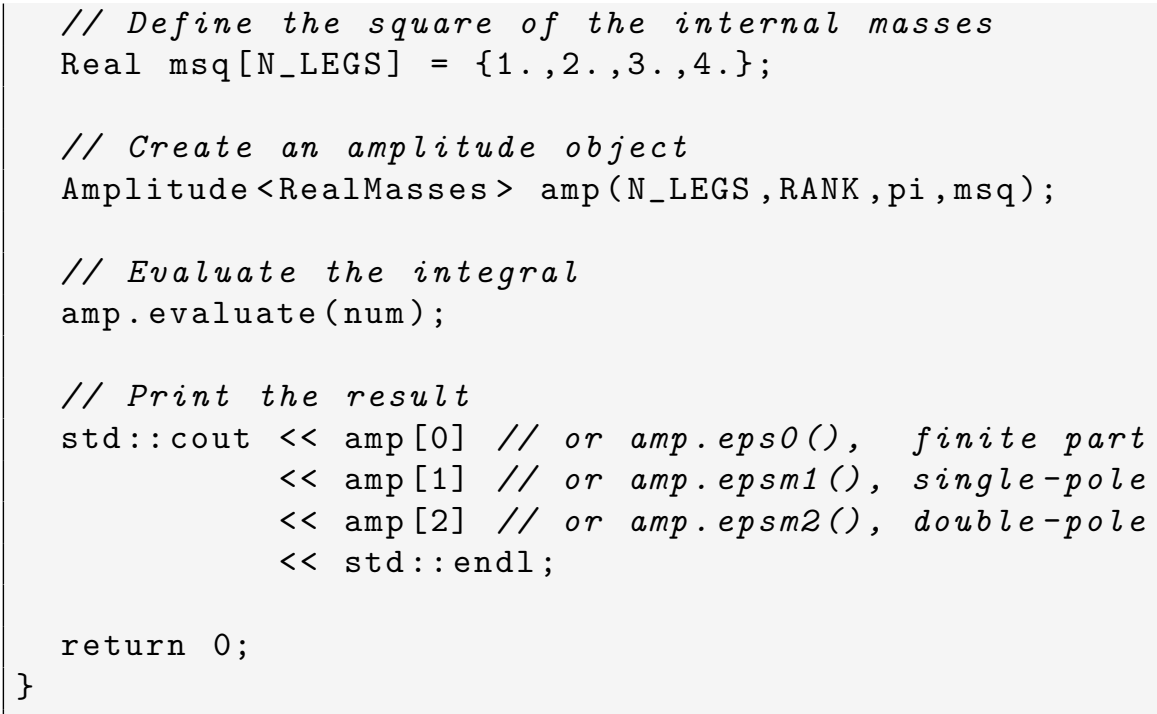

In the example above, after specifying some constants, we declare an instance num of the user-defined class Diagram, which we constructed in Section 4.2. Then we give numerical values to the reference vectors $v_{i}$ appearing in the numerator definition of Eq. (40) as well as in the analytic expression given in the corresponding FORM file. This defines our numerator.

Next we randomly generate a phase-space point, by creating a Rambo object and calling its method getMomenta which fills the array $\mathrm{k}$ of external momenta. The phase space generation is a translation in $\mathrm{C}++$ of the corresponding GoSAM implementation, which in turn is based on the one of Ref. [36]. It is meant to provide an easy way to generate phasespace points in tests which use the library NinJA. Every call of the method getMomenta on the same Rambo object randomly generates a different phase-space point. The code above assumes the external legs to be massless. If the external legs were massive, with masses \{MASS_0, MASS_1, MASS_2, MASS_3\}, we should have generated the phase-space point by passing an array of external masses as third argument to the constructor of the Rambo object, i.e.

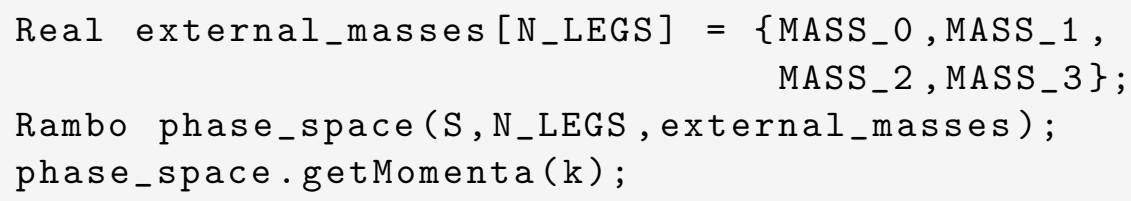

The method getMomenta can optionally take a second parameter, namely a pointer to a Real, into which the weight of the generated phase-space point will be written. In the output, the momenta $\mathrm{k}[0]$ and $\mathrm{k}[1]$ are taken as incoming, while all the others are taken as outgoing. In order to get reproducible results, one can set the random seed with the class method setSeed, which takes an integer as input.

The momenta $p_{i}$ are then defined according to Eq. (40). Arithmetic operations between momentum types work as one would expect. After specifying the square of the internal masses, we create an Amplitude<RealMasses> object amp, whose method evaluate computes the integral with the numerator specified in its argument. This adds the computed integral to the total result stored internally by amp, which can then be accessed either with the methods eps0,epsm1,epsm2 or with the subscript operator "[]" as the example shows. 


\subsubsection{The Amplitude class}

The Amplitude template class is the main class of the NINJA library and its method evaluate computes a one-loop integral. The method takes as input an object of a class derived from ninja: : Numerator, which provides a generic interface to the methods defined by each Laurent expansion. The template parameter of the Amplitude class is the type of the internal masses. Allowed types are: RealMasses, ComplexMasses and Massless. The methods needed for the evaluation of the amplitude are instantiated for all these three types in the compiled code of the library.

Instantiation In the example above, we showed how to instantiate an Amplitude object passing to its constructor the number of external legs, the rank of the numerator, the momenta $p_{i}$ and the squared masses $m_{i}^{2}$ of the loop denominators (Eq. (2)). There we assumed the internal masses to be real. For the complex-masses case and the massless case, the relevant part of the source would have looked like

// Complex masses

Complex msq[N_LEGS $]=\{\ldots\}$;

Amplitude<ComplexMasses > amp (N_LEGS, RANK, pi,msq);

amp.evaluate (num);

and

// Massless (msq does not need to be specified here)

Amplitude<Massless > amp (N_LEGS,RANK, pi);

amp.evaluate (num);

respectively. The Amplitude class also has a default constructor, as well as methods which allow to set or change the kinematics, the (squared) internal masses, the number of legs or the rank of the numerator to be evaluated, as in the following

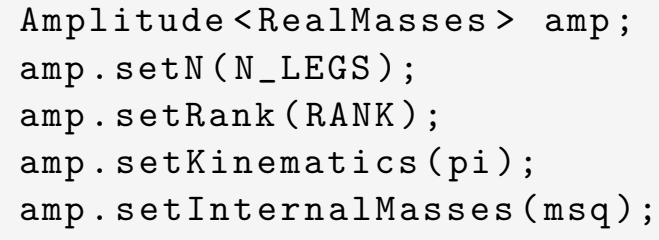

More in detail, the methods setKinematics and setInternalMasses set a data member which is a pointer to the array of momenta (pi) and internal masses (msq) to be used respectively. A call of one of these methods copies the pointer given as input into the corresponding data member. The user should thus make sure that the pointed data will exist in memory until the end of the execution of the evaluate method.

Renormalization scale Another important setting is the renormalization scale $\mu_{R}^{2}$ to be used. This is equal to 1 by default, and it only affects the computation of the Master Integrals. It can be set as in the following example

// takes the square of the scale

amp.setRenormalizationscale $(50 * 50)$; 
The S-matrix An optional parameter which can be set by the user is a matrix of kinematic invariants, which we call S-matrix. This is defined in NinJA by

$$
s_{i j}=\left(p_{i}-p_{j}\right)^{2}
$$

where $p_{i}$ are the momenta appearing in Eq. (2). When this is specified by the user, the computation of the Master Integrals might be more accurate. This can be particularly useful in the presence of infrared singularities, which otherwise might not be detected by the integral library in use. The S-matrix can be declared either by an SMatrix object or by a simple $n^{2}-$ dimensional array, where $n$ is the number of loop denominators. In the simple example given in Section 4.3.1, using the definitions of Eq. (40) in Eq. (41), for massless external momenta $k_{i}$ we could have specified the following S-matrix

$$
\left(s_{i j}\right)=\left(\begin{array}{cccc}
0 & 0 & 2\left(k_{0} \cdot k_{1}\right) & 0 \\
0 & 0 & 0 & -2\left(k_{0} \cdot k_{3}\right) \\
2\left(k_{0} \cdot k_{1}\right) & 0 & 0 & 0 \\
0 & -2\left(k_{0} \cdot k_{3}\right) & 0 & 0
\end{array}\right)
$$

either with

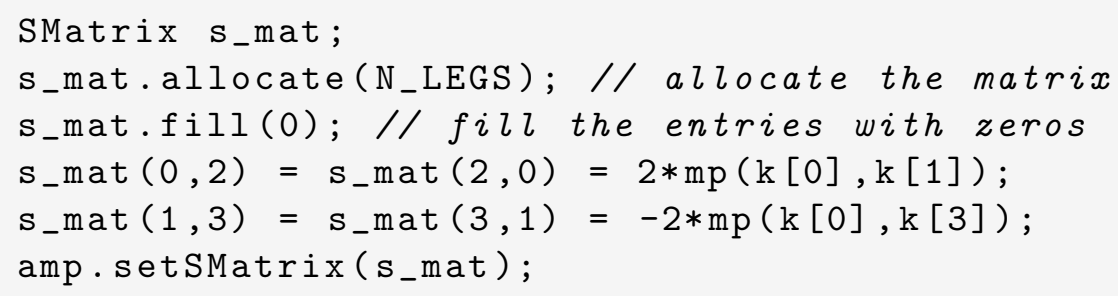

or with

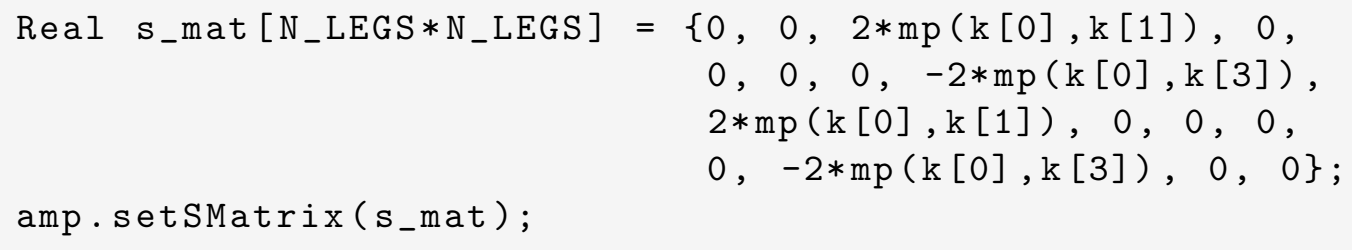

We recommend to specify the S-Matrix whenever possible, and in particular when infrared singularities are present. As an alternative to writing it explicitly, one could use the method

SMatrix \&

SMatrix : fillFromKinematics (const RealMomentum pi[],

Real ir_threshold $=0$ );

before each call of evaluate. This will automatically compute the matrix from the momenta $p_{i}$, but it will set to zero all the matrix elements which are smaller than ir_threshold.

It can be worth pointing out that an Amplitude object, similarly to the case of the internal masses and the kinematics, only stores as data member a pointer to the S-matrix (s_mat) to be used.

Stopping the reduction earlier There are cases where lower-point residues do not contribute to the final result for the integral (see e.g. the example in Section 5.3). If the user knows that $k$-point residues with $k<$ MIN_CUT will not contribute to an amplitude, this information can be passed to NinJA through the setCutStop method, as in this example 
amp.setCutStop (MIN_CUT);

which will tell NiNJA to stop the reduction right after the evaluation of the residues of $k$-ple cuts with $k=$ MIN_CUT.

Master Integrals Each instance of an Amplitude object can in principle use a different library of Master Integrals. The library to be used can be specified with the method setIntegralLibrary as in the following example

Amplitude<RealMasses > amp;

amp.setIntegralLibrary (loop_tools);

If an integral library is not set explicitly for an amplitude object, the instance will use the one which is the default at the time of its creation. The default library will be ONELoop if enabled during configuration, otherwise it will be LoopTools. If none of the two is enabled, Ninja can still be used, but a different library of Master Integrals should be specified at run-time (more details about the implementation of the corresponding interface are given in Appendix C). The function setDefaultIntegralLibrary can be used to change the default integral library. Assuming both OnELOOP and LOOPTOOLS have been enabled, the user can change the default as in the following example

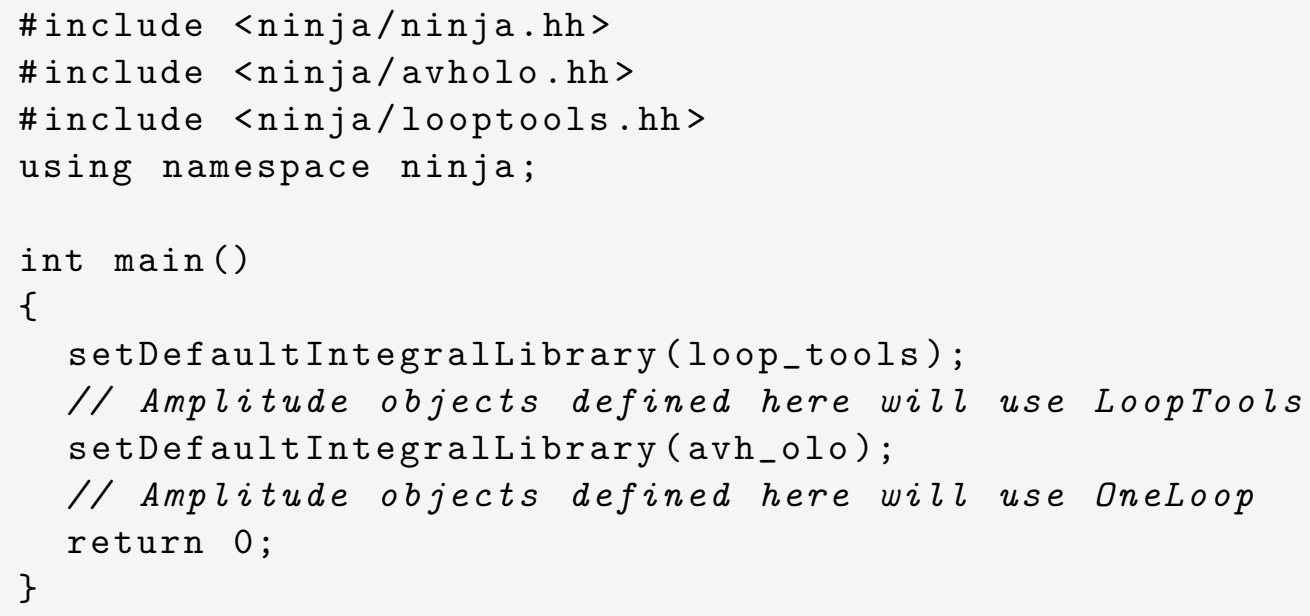

Disabling the $\mu^{2}$ expansion The user can choose to avoid using the $\mu^{2}$ expansion for the boxes with

amp.useMuExpansion (false);

In this case the method muExpansion of the numerator class does not need to be provided. However, this would increase the number calls of the numerator method evaluate and it would also require the computation of pentagons. Given the simplicity of the $\mu^{2}$ expansion, disabling it is therefore not recommended, unless it is done for debugging purposes.

Evaluation of the integrals As already explained, integrals are computed by calling the method evaluate. This has the following prototype 
template <typename MassType>

int Amplitude<MassType >: : evaluate(Numerator \& num);

and takes as input the numerator of the integrand, which must be an instance of a class inherited from ninja: :Numerator. It returns an integer value which depends on the results of the internal tests which NinJA can optionally perform during the computation (see Section 4.3.3). By default no test is performed and the return value can be safely ignored. In general, the return value will be equal to

Amplitude<MassType $>$ : TEST_FAILED if any of the performed tests failed

Amplitude<MassType $>$ : :SUCCESS otherwise.

Each call of the method evaluate adds the computed integral to the total result stored internally by the instance, allowing thus to easily sum different integrals with a sequence of calls of the evaluate method. The total result can then be accessed either with the methods eps0,epsm1,epsm2 or with the subscript operator "[]" as we illustrated in the simple test described in this section. The result can also be quickly reset to zero, by calling the reset method,

amp.reset ();

The finite term is given by the sum of the cut-constructible part and the rational part, but the two can also be accessed separately with

Complex cut_constr_part = amp.getCutConstructiblePart ();

Complex rational_part = amp.getRationalPart ();

\subsubsection{Global settings}

By default NinJa tries to compute a minimal set of coefficients during the reduction, i.e. those which are needed for the determination of the final integrated result. These are only a subset of the ones which are required for the full reconstruction of the integrand decomposition of Eq. (5). Indeed the computation of spurious coefficients is skipped whenever possible, i.e. whenever these do not enter the coefficient-level subtractions needed for lower-point residues, as in the case of spurious coefficients of pentagons, boxes and tadpoles. NinJA also entirely skips the computation of residues whose non-spurious coefficients would multiply scaleless integrals.

For debugging purposes, the user can however ask NinJA to perform some tests on the quality of the reconstruction of the integrand, or print some information about the ongoing computation. These operations might require the computation of a larger set of coefficients.

There are two kinds of tests which NinJA can perform: global tests and local tests. The global $\mathcal{N}=\mathcal{N}$ test $[6,37$ checks that the following equality, which follows from Eq. (5), is valid

$$
\mathcal{N}\left(q, \mu^{2}\right)=\sum_{k=1}^{5} \sum_{\left\{j_{1}, \ldots, j_{k}\right\}} \Delta_{i_{1} \cdots i_{k}} \prod_{h \neq i_{1}, \ldots, i_{k}} D_{h} .
$$


Another global test, which can be performed when the rank $r$ of the numerator is equal to the number of external legs $n$, is the so-called power-test introduced in Ref. [14]. This consists in checking that the sum of all the spurious tadpole coefficients is vanishing,

$$
\sum_{i_{1}=0}^{n-1} \sum_{k=0}^{4} c_{k}^{\left(i_{1}\right)}=0 .
$$

Finally, NinJa can perform local $\mathcal{N}=\mathcal{N}$ tests on quadruple, triple, double and single cuts. These will check the validity of Eq. (43) on values of the loop momenta corresponding to a given $k$-ple cut, and they can be useful in order to pinpoint the multiple cut where an error or an instability has occurred.

By default Ninja does not perform any internal test. The tests to be performed during the execution of the method evaluate can be set using the function

void setTest(unsigned flag);

where the parameter flag can be any of the following:

Test: : NONE no test is performed

Test: : ALL all tests are performed

Test: :GLOBAL global tests are performed

Test : :LOCAL_k with $k \in\{1,2,3,4\}$, local tests on $k$-ple cuts are performed

Test: :LOCAL all local tests are performed

or any combination of these. Different flags can be combined with the bitwise OR operator "I". For instance, the following command will ask NinJA to perform global tests and local tests on double cuts

setTest (Test: : GLOBAL | Test::LOCAL_2);

The $\mathcal{N}=\mathcal{N}$ tests will check whether the numerator $\mathcal{N}_{\text {rec }}$ reconstructed by evaluating the r.h.s. of Eq. (43) is equal to the numerator $\mathcal{N}_{\text {eva }}$, obtained by a direct evaluation through the evaluate method of the numerator class, up to a given tolerance. More explicitly, it checks if

$$
\left|\frac{\mathcal{N}_{\text {rec }}-\mathcal{N}_{\text {eva }}}{\mathcal{N}_{\text {eva }}}\right|<\delta_{\text {tol }}
$$

where the threshold $\delta_{t o l}$ is $10^{-5}$ by default, but it can specified by the user through the function

void setTestTolerance(Real test_tolerance);

As explained in Section 4.3.2, the return value of the method evaluate of the Amplitude class can be used in order to check whether any performed test has failed. These tests have been implemented for debugging purposes, and they are not meant as an estimate of the accuracy of the total result. Indeed, there are cases where a numerical instability might cause a test to fail while having negligible effects on the total amplitude. The accuracy of the result can be better estimated by means of the scaling test proposed in Ref. [22] or the rotation test described in Ref. [27]. 
Another global option which can be set is the verbosity, i.e. the amount of information printed during the evaluation of an integral. By default nothing is printed during a computation. The setting can be controlled by calling the function

void setVerbosity (unsigned flag);

where possible values of the parameter flag can be

Verbose: : NONE nothing is printed

Verbose: :ALL everything is printed (equivalent to the combination of all the other options)

Verbose: :GLOBAL_TEST the result of global tests are printed, when performed

Verbose : :LOCAL_TEST_ $k$ with $k \in\{1,2,3,4\}$, the result of local tests on $k$-ple cuts are printed when performed

Verbose: :LOCAL_TESTS the result of all performed local tests is printed

Verbose: :TESTS the result of all performed tests is printed

Verbose: $\mathrm{C} k$ with $k \in\{1,2,3,4,5\}$, the value of the coefficients of the computed $k$-point residues is printed

Verbose: :COEFFS the value of all the computed coefficients is printed

Verbose: :RESULT the partial result of every call of the evaluate method is printed

Verbose: :INTEGRALS the value of the Master Integrals is printed.

Similarly to the options controlling the performed tests, any combination of the flags above can be specified using the bitwise OR operator "I". As an example, the following instruction will ask NinJA to print the value of the triangle coefficients, and the result of the current integral

setVerbosity(Verbose::C3 | Verbose::RESULT);

When not specified otherwise, NinJA will print everything to standard output. Any other output stream can be set, as in the following example

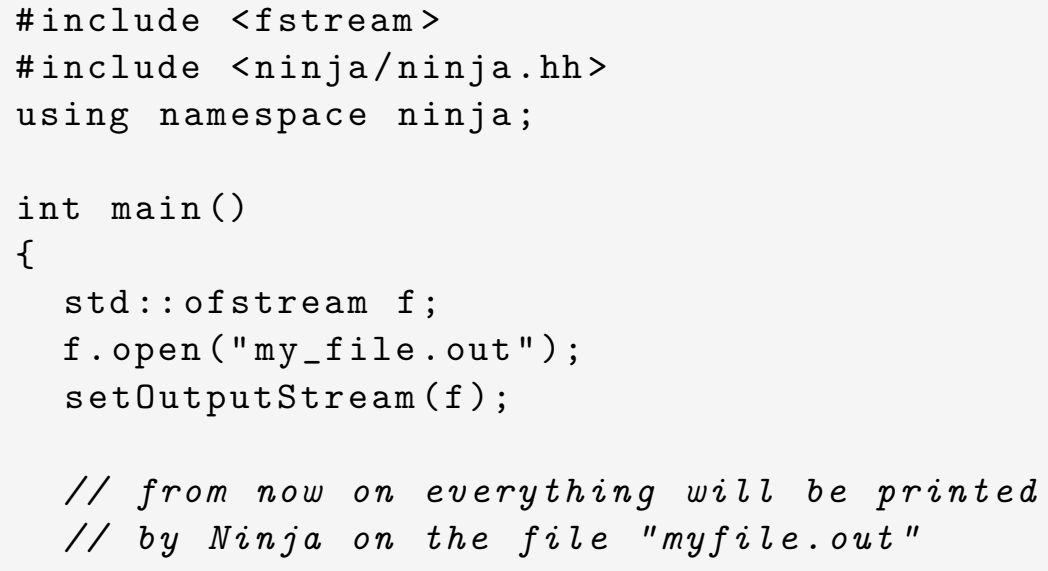




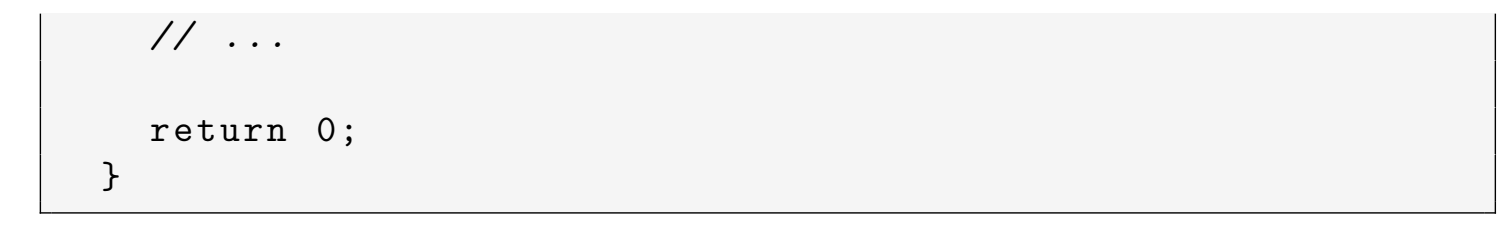

\section{Examples}

In this section we give a description of the examples which are distributed with the library. In order to compile the corresponding executables, one can use the command

make examples

either in the root directory or in the examples directory. These examples are meant to provide a more detailed description of the usage of the library in several kinds of problems which involve the computation of one-loop integrals. More involved computations performed with the help of NinJA have been presented in Ref.s [26,27]. In Ref. [27] one can also find a systematic discussion on the performance and the numerical stability of the library in the computation of scattering amplitudes characterized by high complexity.

Every example presented in this paper has been generated with the help of the package NinjanumGen, which is distributed with Ninja and is described in Appendix A. The package can be used both as a script and as a PYTHON module. For each example, we include in the distribution

- the Form file (with extension .frm) containing the analytic expression of the numerator which is used as input

- a SHELl script (with extension .sh) with the command we used for the generation of the numerator class methods

- a Python script (with extension .py) which achieves the same by importing the ninjanumgen module

- the $\mathrm{C}++$ source files (with extension .cc) and headers files (with extension .hh) defining the numerator and its methods, as well as a test program.

\subsection{Simple Test}

The first simple example has already been extensively described in Section 4, in order to illustrate the basic usage of the library. The numerator class is defined in the header file mynum.hh, the source file generated by NINJANUMGEN is mynum.cc, while the source file with the main function is simple_test.cc.

\section{$5.2 \quad$ Four-photon helicity amplitudes}

In this example we consider a four-photon amplitude $[38,39]$ and we describe the usage of NinJa for the definition of polarization vectors and other spinor objects which are needed for the evaluation of the numerator. 
The integrand of a diagram contributing to a four-photon amplitude is given by

$$
\begin{aligned}
\mathcal{I} & =\frac{\mathcal{N}\left(q, \mu^{2}\right)}{D_{0} D_{1} D_{2} D_{3}} \\
\mathcal{N}\left(q, \mu^{2}\right) & =-\operatorname{Tr}\left(\left(\bar{l} / 1+m_{f}\right) \epsilon_{1}\left(\bar{l}_{2}+m_{f}\right) \epsilon_{2}\left(\bar{l}_{3}+m_{f}\right) \epsilon_{3}\left(\bar{l}_{0}+m_{f}\right) \epsilon_{0}\right) \\
D_{i} & =\bar{l}_{i}^{2}-m_{f}^{2}
\end{aligned}
$$

where $m_{f}$ is the mass of the fermion propagating in the loop, and the momenta $\bar{l}_{i}$ are defined by

$$
\bar{l}_{0}=\bar{q}, \quad \bar{l}_{1}=\bar{q}+k_{0}, \quad \bar{l}_{2}=\bar{q}+k_{0}+k_{1}, \quad \bar{l}_{3}=\bar{q}-k_{4} .
$$

For simplicity we have assumed the four photons to be all incoming, i.e. $k_{0}, k_{1}, k_{2}, k_{3} \rightarrow 0$. The extra-dimensional components $\vec{\mu}$ of the loop momentum satisfy the (anti-)commutation relations

$$
\{\not p, \not \mu\}=0, \quad\{\mu, \not \mu\}=-\mu^{2},
$$

for any four-dimensional momentum $p$. This allows to work out the extra-dimensional algebra and rewrite the numerator in terms of four-dimensional spinor products between the polarization vectors $\epsilon_{i}$, such as $\left\langle\epsilon_{i} \epsilon_{j}\right\rangle$ and $\left[\epsilon_{i} \epsilon_{j}\right]$, and scalar products involving the four-dimensional momenta $q, k_{i}$, and momenta $e_{i j}$ defined by

$$
e_{i j} \equiv \frac{\left\langle\epsilon_{i} \gamma^{\mu} \epsilon_{j}\right]}{2}
$$

The Form package SpInNey [40] can help in this kind of algebraic operations. The final expression can be found in the FORM file 4photons.frm of the directory examples of the distribution.

NinJa includes a small library for massless spinors, which is used internally for building the bases of momenta corresponding to each residue. This can also be useful for defining polarization vectors and other spinor-related objects. The spinor library can be used by including the header file ninja/spinors.hh in the source and linking the program with the Ninja library. Polarization vectors can be defined with

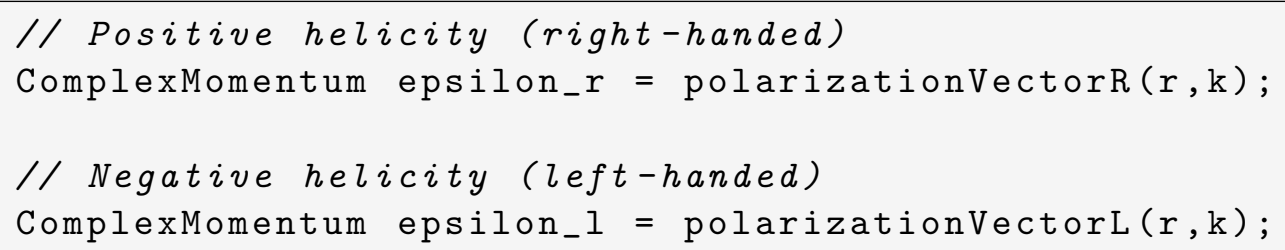

where $r$ is an arbitrary reference momentum and $\mathrm{k}$ is the momentum of the corresponding photon (or gluon). These functions assume the momenta to be incoming, while for outgoing momenta the helicity should be reversed. Angle-bracket and square-bracket spinor products can be computed with the functions spaa and spbb respectively. If $\mathrm{k}$ is a (real or complex) massless momentum, the corresponding spinor spinor_k can be defined as

$$
\text { Spinor spinor_k = Spinor }(k) \text {; }
$$


and can be used as input parameter for the functions described above. This turns out to be more efficient when several spinor-related operations need to be performed on the same momentum. Instances of the class Spinor can also be used in order to define vectors as in Eq. (49), using the following function

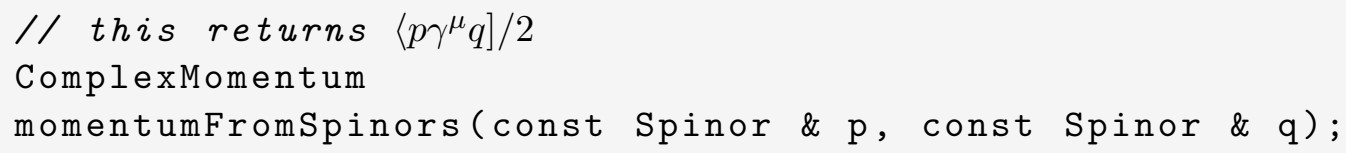

In the header file 4photons_num.hh we define a numerator class FourPhotons containing, as private data members, the values of all the momenta and spinor products appearing in the integrand. The numerator methods have been generated with NinJANumGEN and written in the file 4photons_num.cc. The file 4photons_init.cc contains the implementation of an init method which initializes the data members using the spinor-related operations described above, while 4photons.cc contains a simple test. In this test, the mass of the fermion is complex, thus it can have a width. The results have been compared with the ones in Ref [39] as well as with a similar computation performed with SAMURAI for several choices of the external helicity states and the fermion mass. In Figure 1 we show a typical output for this example.

\subsection{Six-photon helicity amplitudes}

In this example we consider six incoming photons $[37,41-46]$. This is a non-trivial case where the setCutStop method of an Amplitude class can make the computation more efficient when lower point cuts do not contribute to the total result.

A generic six-photon diagram has an integrand of the form

$$
\begin{aligned}
\mathcal{I} & =\frac{\mathcal{N}\left(q, \mu^{2}\right)}{D_{0} D_{1} D_{2} D_{3} D_{4} D_{5}} \\
\mathcal{N}\left(q, \mu^{2}\right) & =-\operatorname{Tr}\left(\bar{l}_{1} \epsilon_{1} \bar{l}_{2} \epsilon_{2} \bar{l}_{3} \epsilon \phi_{3} \bar{l}_{4} \epsilon_{4} \bar{l}_{5} \epsilon t_{5} \bar{l}_{0} \epsilon_{0}\right) \\
D_{i} & =\bar{l}_{i}^{2}
\end{aligned}
$$

where we assumed the fermion running in the loop to be massless. The momenta $\bar{l}_{i}$ are defined by

$$
\begin{array}{lll}
\bar{l}_{0}=\bar{q}, & \bar{l}_{1}=\bar{q}+k_{0}, & \bar{l}_{2}=\bar{q}+k_{0}+k_{1}, \\
\bar{l}_{3}=\bar{q}+k_{0}+k_{1}+k_{2}, & \bar{l}_{2}=\bar{q}-k_{4}-k_{5}, & \bar{l}_{3}=\bar{q}-k_{5} .
\end{array}
$$

One can work out the algebra, define the corresponding spinor products and vectors, and generate the input for NinJA in the same way as for the four-photons case. One can also check that the terms proportional to $\mu^{2}$ in the final expression for the integral vanish upon integration. Therefore, we can perform the simplification $\bar{l}_{i} \rightarrow l_{i}$, or equivalently $\mu^{2} \rightarrow 0$, in the numerator. Moreover, one can exploit the knowledge that only the cut-constructible contributions of boxes and triangles contribute to the total result, hence we can ask NINJA to stop the reduction at triple cuts with

$$
\text { amp.setCutStop (3); }
$$

and remove the rational part from the result with 


\begin{tabular}{ccccc}
\hline \hline PARTICLE & $E$ & $p_{x}$ & $p_{y}$ & $p_{z}$ \\
\hline$k_{0}$ & 7.0000000000000000 & 0.0000000000000000 & 0.0000000000000000 & 7.0000000000000000 \\
$k_{1}$ & 7.0000000000000000 & 0.0000000000000000 & 0.0000000000000000 & -7.0000000000000000 \\
$k_{2}$ & -6.9999999999999964 & -6.1126608202785198 & 0.8284979592001092 & -3.3089226083172685 \\
$k_{3}$ & -7.0000000000000027 & 6.1126608202785278 & -0.8284979592001093 & 3.3089226083172703 \\
\hline \hline
\end{tabular}

\begin{tabular}{|c|c|c|c|}
\hline \multicolumn{4}{|c|}{ 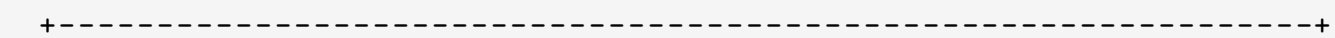 } \\
\hline 1 & & I \\
\hline 1 & & & I \\
\hline 1 & \multicolumn{2}{|c|}{ Ninja - version 1.0 .0} & I \\
\hline I & \multicolumn{3}{|c|}{ Author: Tiziano Peraro } \\
\hline I & \multicolumn{2}{|c|}{ Author: Tiziano Peraro } & I \\
\hline 1 & \multirow{2}{*}{\multicolumn{2}{|c|}{ Based on: }} & i \\
\hline 1 & & & I \\
\hline I & \multicolumn{3}{|c|}{ P. Mastrolia, E. Mirabella and T. Peraro, } \\
\hline i & \multicolumn{3}{|c|}{ "Integrand reduction of one-loop scattering amplitudes } \\
\hline I & \multicolumn{3}{|c|}{ through Laurent series expansion," } \\
\hline i & \multicolumn{2}{|r|}{ JHEP 1206 (2012) 095 [arXiv:1203.0291 [hep-ph]]. } & i \\
\hline I & \multirow{2}{*}{\multicolumn{2}{|c|}{ T. Peraro, }} & I \\
\hline 1 & & & I \\
\hline 1 & \multicolumn{2}{|r|}{ "Ninja: Automated Integrand Reduction via Laurent } & I \\
\hline 1 & \multicolumn{2}{|r|}{ Expansion for One-Loop Amplitudes," } & I \\
\hline i & \multicolumn{2}{|c|}{$\operatorname{arXiv}: 1403.1229 \quad[$ hep-ph] } & i \\
\hline \multicolumn{3}{|c|}{ acte- } & I \\
\hline \multicolumn{4}{|c|}{ 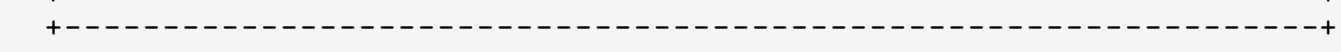 } \\
\hline \multicolumn{4}{|c|}{ Finite : $\quad(-0.184034,-0.16765)$} \\
\hline \multicolumn{4}{|c|}{ Abs. val.: 0.248948} \\
\hline \multicolumn{4}{|c|}{ Single pole: $(-3.73035 e-13,3.98348 e-13)$} \\
\hline \multicolumn{4}{|c|}{ Double pole: $(0,0)$} \\
\hline
\end{tabular}

Figure 1: Phase space point and output for the example in 4photons.cc. It shows the computed finite part and poles of an all-plus four-photon helicity amplitude, using a complex fermion mass $m_{f}=10.0-1.0 i$.

amp.onlyCutConstructible();

which will make the computation more efficient (in the example implemented here, the runtime is reduced by about $33 \%$ ).

In the file 6photons.cc we call the method evaluate on all the independent permutations of the external legs, generated at run-time with the function std: : next_permutation of the $\mathrm{C}++$ standard library. The results have been compared with the ones in Ref.s $[45,46]$ as well as with a similar computation performed with SAMURAI for several helicity choices.

\subsection{Five-point diagram of $g g \rightarrow H t \bar{t}$}

With this example, we discuss a possible strategy for the generation of the input needed by NinJA which can be suited for more complex computations where an efficient evaluation of the numerator methods at run-time can be important.

We consider the one-loop integral defined by the diagram depicted in Figure 2, contributing 
to the 5 -point helicity amplitude $g\left(k_{1},-\right), g\left(k_{2},-\right) \rightarrow H\left(k_{3}\right), t\left(k_{4},+\right), \bar{t}\left(k_{5},-\right)$. The analytic

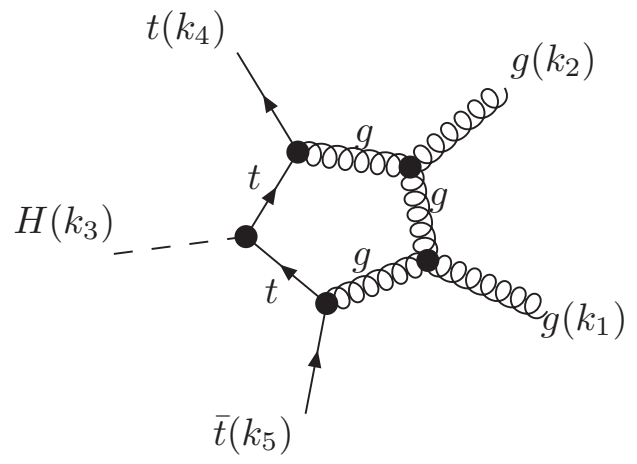

Figure 2: Diagram contributing to $g g \rightarrow H t \bar{t}$. This picture has been generated using GoSAM.

expression for the integrand of this example, which can be worked out from the Feynman rules of the Standard Model, has been generated with the help of the package GoSAM and can be found in the Form file ttbarh.frm. This already contains some abbreviations which are independent from the loop momentum $\bar{q}$ of the diagram. At run-time, these $\bar{q}$-independent abbreviations are computed only once per phase space point, making thus the evaluation of the numerator and its expansions more efficient. This analytic expression is processed by NinJANumGen which produces the numerator expansions. We also add to the numerator class TTbarHDiagram an init method which uses the spinor library described in Section 5.2 in order to compute the relevant spinor products and polarization vectors, as well as the abbreviations which do not depend on the loop momentum. These are stored as private data members of the class. For simplicity, our result neglects the coupling constants and an overall color factor.

Even though we considered a single diagram and a specific helicity choice, this example illustrates a general strategy for the generation of an analytic numerator expression which is suited for the numerical evaluations performed by integrand-reduction algorithms like the one implemented in the library NinJA. The full amplitude for this process has been computed in Ref.s [47-51], while an additional jet has recently been added to the final state in Ref.s [26,27] where NinJa has been used for the reduction of the corresponding integrands generated by GoSAM.

\subsection{Higher-rank example}

In this example we show how NINJA can be used in order to compute integrals whose rank is higher than the number of loop denominators. This simple test is similar to the example presented in Ref. 4, hence we will describe each step as in the previous case. We define a 
5 -point amplitude of rank 6 , with kinematics $k_{0}, k_{1} \rightarrow k_{2}, k_{3}, k_{4}, k_{5}$ and integrand

$$
\begin{aligned}
\mathcal{I} & =\frac{\mathcal{N}\left(q, \mu^{2}\right)}{D_{0} D_{1} D_{2} D_{3} D_{4}} \\
\mathcal{N}\left(q, \mu^{2}\right) & =\prod_{i=0}^{2}\left(\left(q \cdot v_{2 i}\right)\left(q \cdot v_{2 i+1}\right)+\mu^{2}\left(v_{2 i} \cdot v_{2 i+1}\right)\right) \\
D_{i} & =\bar{l}_{i}^{2}-m_{i}^{2}
\end{aligned}
$$

in terms of the reference vectors $v_{i}(i=0, \ldots, 5)$ and the momenta $\bar{l}_{i}$ running into the loop

$$
\bar{l}_{0}=\bar{q}, \quad \bar{l}_{1}=\bar{q}+k_{0}, \quad \bar{l}_{2}=\bar{q}+k_{0}+k_{1}, \quad \bar{l}_{3}=\bar{q}+k_{3}+k_{4}, \quad \bar{l}_{4}=\bar{q}+k_{4} .
$$

We follow the same steps outlined in Section 4. With NinjaNumGEN we generate the methods for NinJA. After writing the integrand in the Form file mynumhr.frm we call the script with the command

$$
\text { ninjanumgen mynumhr.frm --nlegs } 5 \text {--rank } 6 \text {-o mynumhr.cc }
$$

which creates the file mynumhr.cc and a template for the header mynumhr.hh. Once again, we define the vectors $v_{i}$ as public members of the numerator class Diagram, by inserting

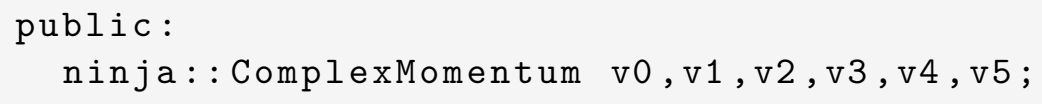

in the class definition. A possible test program can be almost identical to the one we showed in Section 4.3.1, with obvious changes in the definition of the rank, the number of external legs and the reference vectors $v_{i}$. This is implemented in the file simple_higher_rank_test.cc. In order to run this example, the user must compile the library with the--enable-higher_rank option, otherwise a call to the evaluate method of an Amplitude object will cause a run-time error.

As one can see, when NinJANumGen is used for the generation of the expansions, the higher-rank case is handled automatically without any intervention by the user. Besides, the internal higher-rank routines of NINJA will be automatically called whenever the rank $r$ is equal to $n+1$ (where $n$ is the number of loop propagators), while in the public programming interface there is no difference with respect to the normal-rank case.

\subsection{Usage in multi-threaded applications}

In the last examples, we wish to illustrate the possibility of using NinJA in a multi-threaded application. These examples are implemented using POSIX threads, which are a standard in Unix-like operating systems, but adapting them to different programming interfaces for threads (such as OpenMP) should be straightforward.

In order to implement a thread-safe application, one should avoid race conditions which might occur if different threads try to write on the same variables. In particular, one should avoid accessing global variables for writing from different threads. The only global variables used directly by NinJA are those controlling the global options described in Section 4.3.3. As explained in that section, these options are only meant to change the general behavior of the library for debugging purposes (e.g. for checking that the provided numerator methods are correct), while in general the default options should not be changed during a phase-space 
integration, especially when performance is important. Hence, on the side of the NINJA library, there should be no issue and one can safely call the evaluate method from different Amplitude objects in different threads.

During a call of the evaluate method on an Amplitude object, issues might however arise from global variables used by the chosen library of Master Integrals or the numerator methods. As for the numerator methods, all the examples distributed with NINJA define a threadsafe numerator class (more specifically, one can safely call numerator methods from different instances of the class in different threads). This is simply done by using data members of the class instead of global variables, making thus different instances of the same class completely independent.

If the procedures implemented by libraries of Master Integrals are thread-safe, one can therefore use NinJa in multi-threaded applications. As an example, one can use the class AvHOneLoop which, as explained in Section 3.3, wraps routines of the OnELOOP library and adds a cache of computed integrals. The cache is a non-static data member of the class. One can therefore create one instance of this class per thread and assign it accordingly to the Amplitude objects to be evaluated in the same thread. As an example, with

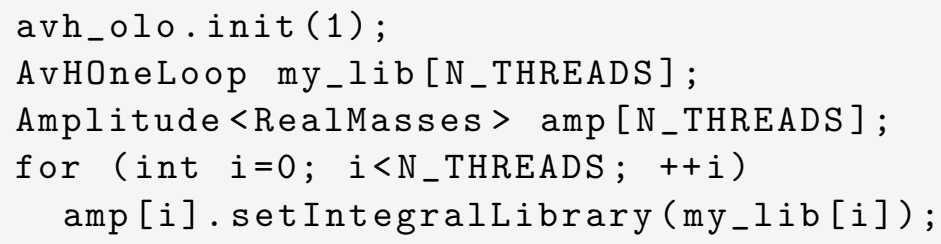

we create N_THREADS amplitude objects whose evaluate method can be safely called in a separate thread (in the first line, we called the init method on the global instance avh_olo defined in the library, in order to allow ONELOOP to perform its global initialization). In this way, different threads will also have an independent cache of Master Integrals. This strategy allows to build a multi-threaded application which uses NinJA for the reduction of one-loop integrals. Recent versions of LoopTOoLs (namely LoopTools-2.10 or later) can also be used in threaded applications, since they have a mutex regulating writing access to the internal cache of integrals.

In the following we discuss the possibility to build a multi-threaded application with NINJA and any other (not necessarily thread-safe) library of Master Integrals. Indeed, even though NinJa has obviously no control over possible issues arising from routines of external libraries, we offer an easy way to work around any potential problem. In this case, there is no general way to ensure that calling routines of the same integral library from different threads will not cause conflicts. However, one can avoid these conflicts by scheduling the calls of the external procedures in such a way that they are never evaluated at the same time from two or more threads. If the computation of the Master Integrals takes only a small fraction of the total run time (which is usually the case when a cache of integrals is present), the effects of this on the performance will in general be reasonably small.

Within NinJA, implementing a scheduled access on the routines used by a library of Master Integrals is straightforward. As explained more in detail in Appendix C, the generic interface used by NinJA in order to call Master Integral procedures, has two methods called init and exit which are evaluated exactly once in each call of the evaluate method, immediately before the computation of the first Master Integral and after the computation of the last Master Integral respectively. Therefore we can use mutexes (such as the ones present the POSIX standard for threads) in order to lock the calls to the Master Integrals in the init 
method and unlock them in the exit method. This makes sure that, between the calls of the init and exit methods, no other thread will use the Master Integral routines, hence avoiding any possible conflict.

In order to make a library of Master Integrals thread-safe, we use the template class ThreadSafeIntegralLibrary, which is included in the distribution. This automatically wraps an existing class derived from IntegralLibrary and adds to it a mutex that schedules the calls to the Master Integrals as explained above. As an example, defining a thread-safe version of a generic library BaseLibrary can be simply achieved with

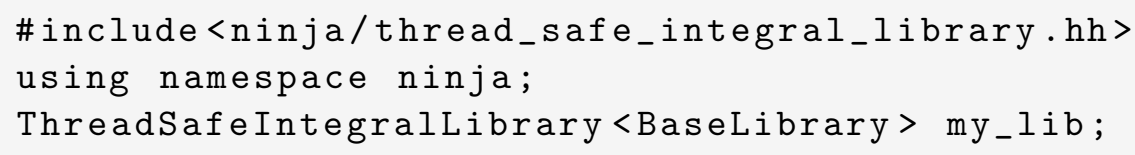

which defines a new interface my_lib that can be made the default by calling

setDefaultIntegrallibrary (my_lib);

before any thread is created (alternatively, we could call the setIntegralLibrary method on each Amplitude object, either outside or inside the threads).

In the files thread_4photons.cc and thread_6photons.cc we repeat the examples of the four- and six-photons amplitudes, but this time we compute several phase-space points in parallel on different threads. As mentioned before, we do not need to implement other numerator classes, since the ones described in Sections 5.2 and 5.3 can be safely used in multi-threaded applications. In the source files, we implement both the approaches described in this section. The preprocessor will select the former if the ONELoOP interface has been enabled and the latter otherwise. The multi-threaded examples can be compiled with

make thread-examples

if at least one between the OnELoOP and LoOpTools libraries was enabled during configuration and your system supports POSIX threads.

A complete discussion on the implementation of multi-threaded applications for doing phenomenology at one-loop is beyond the purposes of this paper. Moreover, a detailed assessment of possible advantages of this approach would generally depend on the generator of the integrands and the phase space integration. In these examples we showed that the methods implementing the reduction via Laurent expansion in NINJA can be safely used in multi-threaded programs.

\section{Conclusions}

We presented the public $\mathrm{C}++$ library NinJA which implements the Integrand Reduction via Laurent Expansion method for the computation of one-loop amplitudes in Quantum Field Theories. The main procedures of the library take as input the numerator of the integrand and some parametric expansions of the same, which can be generated with the help of the simple Python package NinjaNumGen included with the distribution. The expansions of the integrand on the multiple cuts are computed semi-numerically at run-time, via a simplified polynomial-division algorithm. Some of the coefficients of the Laurent expansions are thus identified with the ones which multiply the Master Integrals. The algorithm is light and proved to have good performance and numerical stability, hence it is suited for applications 
to complex one-loop processes, characterized by either several external legs or several mass scales.

We described the usage of the library, in particular the generation of the input, the calls of the procedures for the reduction, and the interface to libraries of Master Integrals. This information can be used in order to interface the library with existing one-loop packages. We thus expect that NinJA will be useful for future computations in high-energy physics, especially for those involving more complex processes.

\section{Acknowledgments}

The author thanks all the other members of the GoSAM collaboration for the common development of a one-loop package which could be interfaced with NinJA, and especially Pierpaolo Mastrolia, Edoardo Mirabella and Giovanni Ossola for innumerable discussions and exchanges. The author also thanks Thomas Hahn for his support with LoopTools and comments on the draft. This work was supported by the Alexander von Humboldt Foundation, in the framework of the Sofja Kovalevskaja Award Project "Advanced Mathematical Methods for Particle Physics", endowed by the German Federal Ministry of Education and Research.

\section{Appendix A The Python package NinjaNumGen}

The reduction procedures implemented in NinJA take as input a class derived from the abstract class ninja: : Numerator. This must implement the required expansions in the corresponding methods. If the analytic expression of the numerator can be provided by the user, the source code for the methods can be automatically generated with the help of the simple Python package NinjaNumGen, which is distributed with the library and can be installed as explained in Section 4.1. The package can be used both as a script or as a module within PYTHON.

In Section 4.2 we already gave a simple example of its usage as a script. As explained there, the user must create a file containing a FORM expression of the numerator of the integrand. The package uses ForM-4 in order to generate the expansions which are needed and produce a $\mathrm{C}++$ source file with the definitions of the corresponding methods. If not already present, an header file with a sketch of the definition of the class will also be created. The user can complete it by adding data members and methods which are specific of this class. Form allows one to define symbols between square brackets (e.g. [symbol_name]), containing characters which otherwise would not be permitted in a declaration. NiNJANuMGEN also allows the usage of such symbols in the expression of the numerator, and it will remove the brackets (which would produce illegal $\mathrm{C}++$ code) when writing the final source files. This gives the user a wider range of possibilities, for instance using symbols which correspond to variable names containing underscores or data members of structures (e.g. with [structure_instance.data_member]).

We first give a few more details about the usage of the package as a script. It is invoked with the command

ninjanumgen --nlegs NLEGS optional-arguments file 
where file is the name of the file which contains the numerator expression and NLEGS is the number of external legs of the loop, which is equal to the number of loop denominators. A description of all the allowed arguments can be obtained with the command

$$
\text { ninjanumgen --help }
$$

and the most important ones are:

--rank RANK, $-r$ RANK rank of the numerator, by default it will be assumed to be equal to the number of external legs of the loop

--diagname DIAGNAME, -d DIAGNAME name of the numerator expression in the FORM file, by default it will be assumed to be Diagram

--cdiagname CDIAGNAME name of the numerator class in the generated $\mathrm{C}++$ files, by default it will be the same as the Form expression

--formexec FORMEXEC the FORM executable, the default is form

--qvar QVAR name of the loop momentum variable $q$ defined in Eq. (4), the default is Q

--mu2var MU2VAR name of the loop variable $\mu^{2}$ defined in Eq. (4), the default is Mu2

--output OUTPUT, -o OUTPUT name of the output source file, the default is ninjanumgen.cc

--header HEADER $\mathrm{C}++$ header file containing the definition of the numerator class: if the file does not exists, one will be created. By default it will have the same name as the output but with .hh extension.

As mentioned, one can also use the package as a PYTHON module (ninjanumgen). This contains a class DiagramExpansion which can be used for the generation of the source code which implements the numerator methods. The input parameters of the constructor of this class roughly correspond to the arguments which can be used in the script. A detailed description can be obtained, after installation, by invoking PYTHON in interactive mode (usually done with the command python) and typing

import ninjanumgen

help (ninjanumgen. DiagramExpansion )

The method writeSource generates the source files. As a simple example, the source for the integrand we defined in Section 4.2 could have been generated within PYTHON with the commands

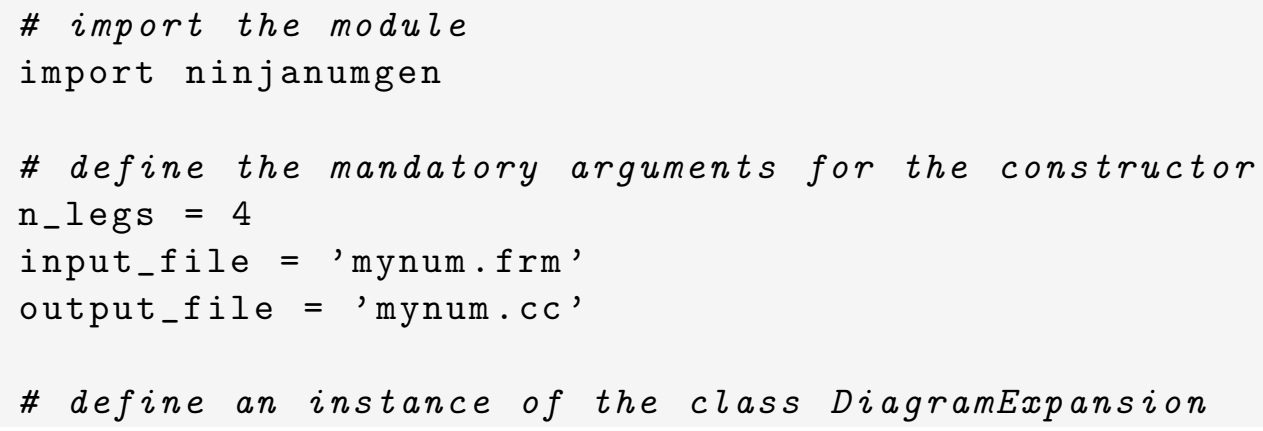




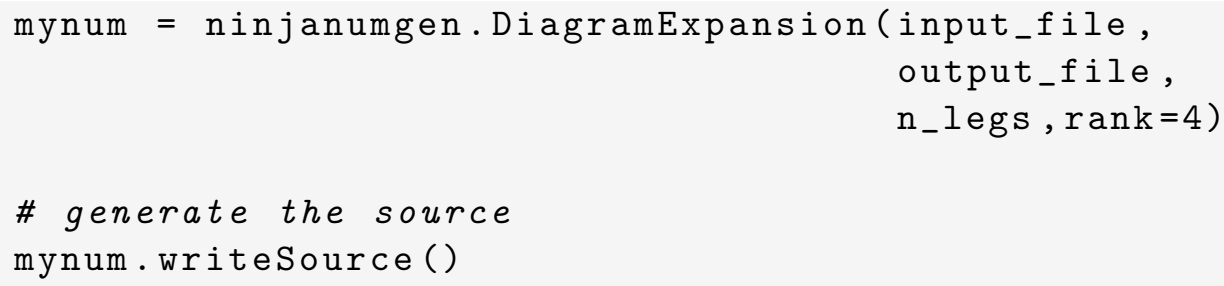

We suggest to look at the PYTHON files in the examples directory for other basic examples.

\section{Appendix B Higher-rank numerators}

As pointed out in Ref. [24], the Laurent expansion method can be generalized to nonrenormalizable and effective theories with higher-rank numerators. In a renormalizable theory, with a proper choice of gauge the rank $r$ cannot be greater than the number $n$ of loop propagators. NinJA, if configured with the--enable-higher_rank option, can also be used for the computation of integrals with $r=n+1$. Here we describe the generalization of the method to the higher-rank case, underlining the points where it differs from the renormalizable case.

In Eq. (14), we gave the most general parametrization of the residues $\Delta_{i_{1} \cdots i_{k}}$ in a renormalizable theory. In the higher-rank case with $r=n+1$, such parametrization is generalized as follows [24]

$$
\begin{aligned}
\Delta_{i_{1} i_{2} i_{3} i_{4} i_{5}}^{(r=n+1)}= & \Delta_{i_{1} i_{2} i_{3} i_{4} i_{5}} \\
\Delta_{i_{1} i_{2} i_{3} i_{4}}^{(r=n+1)}= & \Delta_{i_{1} i_{2} i_{3} i_{4}}+c_{5} \mu^{4} x_{v, 4} \\
\Delta_{i_{1} i_{2} i_{3}}^{(r=n+1)}= & \Delta_{i_{1} i_{2} i_{3}}+\mu^{2}\left(c_{10} x_{4}^{2}+c_{11} x_{3}^{2}\right)+c_{12} x_{4}^{4}+c_{13} x_{3}^{4}+c_{14} \mu^{4} \\
\Delta_{i_{1} i_{2}}^{(r=n+1)}= & \Delta_{i_{1} i_{2}}+\mu^{2}\left(c_{10} x_{1}+c_{11} x_{4}+c_{12} x_{3}\right)+c_{13} x_{1}^{3}+c_{14} x_{4}^{3}+c_{15} x_{3}^{3} \\
& +c_{16} x_{1}^{2} x_{4}+c_{17} x_{1}^{2} x_{3}+c_{18} x_{1} x_{4}^{2}+c_{19} x_{1} x_{3}^{2} \\
\Delta_{i_{1}}^{(r=n+1)}= & \Delta_{i_{1}}+c_{5} x_{2}^{2}+c_{6} x_{1}^{2}+c_{7} x_{4}^{2}+c_{8} x_{3}^{2}+c_{10} x_{2} x_{4}+c_{11} x_{2} x_{3} \\
& +c_{12} x_{1} x_{4}+c_{13} x_{1} x_{3}+c_{14} \mu^{2}+c_{15} x_{3} x_{4} .
\end{aligned}
$$

The generalized integral decomposition is thus

$$
\begin{aligned}
\mathcal{M}^{(r=n+1)}= & \mathcal{M}^{(r=n)}+\sum_{\left\{i_{1}, i_{2}, i_{3}\right\}} c_{14}^{\left(i_{1} i_{2} i_{3}\right)} I_{i_{1} i_{2} i_{3}}\left[\mu^{4}\right] \\
& \left.+\sum_{\left\{i_{1}, i_{2}\right\}}\left\{c_{10}^{\left(i_{1} i_{2}\right)} I_{i_{1} i_{2}}\left[\mu^{2}\left(q+p_{i_{1}}\right) \cdot e_{2}\right)\right]+c_{13}^{\left(i_{1} i_{2}\right)} I_{i_{1} i_{2}}\left[\left(\left(q+p_{i_{1}}\right) \cdot e_{2}\right)^{3}\right]\right\} \\
& +\sum_{i_{1}}\left\{c_{14} I_{i_{1}}\left[\mu^{2}\right]+c_{15}^{\left(i_{1}\right)} I_{i_{1}}\left[\left(\left(q+p_{i_{1}}\right) \cdot e_{3}\right)\left(\left(q+p_{i_{1}}\right) \cdot e_{4}\right)\right]\right\}
\end{aligned}
$$

This higher-rank decomposition has been used for the computation of NLO corrections to Higgs-boson production in association with two [52] and three $[27,53]$ jets. Other libraries which implement the reduction of higher-rank integrals are XSAMURAI [54], which extends the more traditional integrand reduction algorithm of SAMURAI, and GolEm95 [55-57]. 


\section{B.1 Reduction of higher-rank integrands}

While the extension of the Laurent expansion method for the computation of higher-rank 3-point and 2-point residues is straightforward, for 4-point and 1-point residues some further observations are in order. Here we propose a generalization of the Laurent expansion method which allows to efficiently compute the non-spurious coefficients of 4- and 1-point residues without spoiling the nice features of the algorithm, such as the simplified subtractions of higher-point contributions and the diagonal systems of equations. This generalization is not present elsewhere in the literature and has been implemented in the NinJA library.

4-point residues The coefficient $c_{0}$ can be computed exactly as in the renormalizable case. For the coefficient $c_{4}$, one needs instead to keep also the next-to-leading term in the $\mu^{2}$ expansion described before, so that the $d$-dimensional solutions of a quadruple cut, given in Eq. (17), in the asymptotic limit become

$$
q_{ \pm}^{\nu}=-p_{i_{1}}^{\nu}+a^{\nu} \pm \sqrt{\mu^{2}+\beta} v_{\perp}^{\nu} \stackrel{\mu^{2} \rightarrow \infty}{=}-p_{i_{1}}^{\nu}+a^{\nu} \pm \sqrt{\mu^{2}} v_{\perp}^{\nu}+\mathcal{O}\left(\frac{1}{\sqrt{\mu^{2}}}\right)
$$

where it is worth noticing that $a^{\nu}$ can be obtained as the average of the two solutions of the corresponding four-dimensional quadruple cut. In this limit, the expansion of the integrand reads

$$
\left.\frac{\mathcal{N}\left(q, \mu^{2}\right)}{\prod_{j \neq i_{1}, i_{2}, i_{3}, i_{4}} D_{j}}\right|_{q=\sqrt{\mu^{2}} v_{\perp}+a+\mathcal{O}\left(\mu^{-1}\right)}=c_{5} v_{\perp}^{2} \mu^{5}+c_{4} \mu^{4}+\mathcal{O}\left(\mu^{3}\right),
$$

hence the leading term is now the spurious coefficient $c_{5}$, but $c_{4}$ can still be obtained as the next-to-leading term. This can be implemented semi-numerically, by keeping the two leading terms of the expansion of the numerator and performing a polynomial division with respect to the two leading terms in the expansion of the uncut denominators which have the form

$$
\left.D_{h \neq i_{1}, i_{2}, i_{3}, i_{4}}\right|_{q=q_{+}}=d_{h, 0} \sqrt{\mu^{2}}+d_{h, 1}+\mathcal{O}\left(\frac{1}{\sqrt{\mu^{2}}}\right) .
$$

Given the very limited number of terms involved, the division can be implemented very efficiently in a small number of operations. More in detail, if num and den are arrays of length two containing the leading and next-to-leading terms in the expansion of the numerator and a denominator respectively, we can perform the division in place with the commands

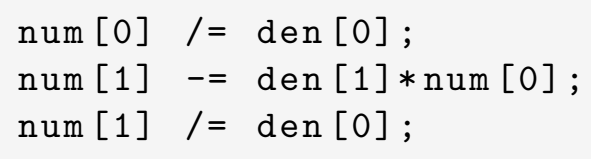

which will have the effect of replacing the entries of num with the ones of the expansion of $\mathcal{N} / D$. We also observe that the computation and the subtraction of pentagons is not needed in the higher-rank case either.

1-point residues On higher-rank 1-point residues $\Delta_{i_{1}}$ we consider $d$-dimensional solutions of the corresponding single cut of the form

$$
q_{+}^{\nu}=-p_{i_{1}}+t e_{1}^{\nu}+\frac{m_{i_{1}}^{2}+\mu^{2}}{2 t\left(e_{1} \cdot e_{2}\right)} e_{2}^{\nu}, \quad q_{-}^{\nu}=-p_{i_{1}}+t e_{3}^{\nu}+\frac{m_{i_{1}}^{2}+\mu^{2}}{2 t\left(e_{3} \cdot e_{4}\right)} e_{4}^{\nu},
$$


in terms of the free variables $t$ and $\mu^{2}$. By taking the $t \rightarrow \infty$ limit of the integrand and the subtraction terms evaluated on these solutions, we obtain an asymptotic polynomial expansion of the form

$$
\begin{gathered}
\frac{\mathcal{N}\left(q_{ \pm}, \mu^{2}\right)}{\prod_{j \neq i_{1}} D_{j}}=n_{0}^{ \pm}+n_{1}^{ \pm} t+n_{2}^{ \pm} t^{2}+n_{3}^{ \pm} \mu^{2}+\mathcal{O}(1 / t) \\
\frac{\Delta_{i_{1} j}\left(q_{ \pm}, \mu^{2}\right)}{D_{j}}=c_{s_{2}, 0}^{ \pm(j)}+c_{s_{2}, 1}^{ \pm(j)} t+c_{s_{2}, 2}^{ \pm(j)} t^{2}+c_{s_{2}, 3}^{ \pm(j)} \mu^{2}+\mathcal{O}(1 / t) \\
\frac{\Delta_{i_{1} j k}\left(q_{ \pm}, \mu^{2}\right)}{D_{j} D_{k}}=c_{s_{3}, 0}^{ \pm(j k)}+c_{s_{3}, 1}^{ \pm(j k)} t+c_{s_{3}, 2}^{ \pm(j k)} t^{2}+c_{s_{3}, 3}^{ \pm(j k)} \mu^{2}+\mathcal{O}(1 / t) .
\end{gathered}
$$

One can check that the non-spurious coefficients of the tadpole are given in terms of the ones of the expansions above by

$$
\begin{aligned}
c_{0} & =n_{0}^{+}-\sum_{j} c_{s_{2}, 0}^{+(j)}-\sum_{j k} c_{s_{3}, 0}^{+(j k)}, \\
c_{14} & =n_{3}^{+}-\sum_{j} c_{s_{2}, 3}^{+(j)}-\sum_{j k} c_{s_{3}, 3}^{+(j k)}, \\
c_{15} & =\frac{2}{\left(e_{3} \cdot e_{4}\right)}\left(n_{3}^{-}-\sum_{j} c_{s_{2}, 3}^{-(j)}-\sum_{j k} c_{s_{3}, 3}^{-(j k)}-c_{14}\right) .
\end{aligned}
$$

\section{B.2 The input}

In the higher-rank case, the muExpansion method of the numerator needs to compute both the leading and the next-to-leading term of the expansion in $\mu^{2}$. The package NinJANumGEN, takes care of this automatically when the specified rank is higher than the number of external legs of the loop. The information in the next paragraph is only needed for a custom implementation of the method without NINJANUMGEN.

The muExpansion method in the higher-rank case should compute the two leading terms of the expansion in $t$ of the numerator, defined by

$$
q^{\nu} \rightarrow t v_{0}^{\nu} t+v_{1}^{\nu}, \quad \mu^{2} \rightarrow t^{2} v_{0}^{2}
$$

with $v_{i} \equiv v$ [i], where $v$ is the array of momenta passed as input parameter. The leading and next-to-leading terms of the expansion should be written in the first two entry of the array pointed by the parameter c, i.e.

$$
\begin{aligned}
& c[0]=\mathcal{N}\left[t^{r}\right] ; \\
& c[1]=\mathcal{N}\left[t^{r-1}\right] ;
\end{aligned}
$$

All the other methods should have instead the same definition described in Section 3.1.

\section{B.3 Higher-rank Master Integrals}

As one can see from Eq. (B.2), in the higher-rank case five new types of integral appear in the final decomposition. They are a 2-point integral of rank 3, a 1-point integral of rank 2, and three more integrals containing $\mu^{2}$ at the numerator which contribute to the rational part of the amplitude. 
NiNJA contains an implementation of all these higher-rank integrals in terms of lowerrank ones. This means that, should the user choose to interface a custom integral library (see Appendix C), these higher-rank integrals would not be needed, although specifying an alternative implementation would still be possible.

All the integrals of Eq. (B.2) which contribute to the rational part of the amplitude have already been computed in Ref. [24]. With our choice of the normalization factor given in Eq. (3), they read

$$
\begin{aligned}
I_{i_{1} i_{2} i_{3}}\left[\mu^{4}\right] & =\frac{1}{6}\left(\frac{s_{i_{2} i_{1}}+s_{i_{3} i_{2}}+s_{i_{1} i_{3}}}{4}-m_{i_{1}}^{2}-m_{i_{2}}^{2}-m_{i_{3}}^{2}\right)+\mathcal{O}(\epsilon) \\
I_{i_{1} i_{2}}\left[\mu^{2}\left(\left(q+p_{i_{1}}\right) \cdot v\right)\right] & =\frac{\left(\left(p_{i_{2}}-p_{i_{1}}\right) \cdot v\right)}{12}\left(s_{i_{2} i_{1}}-2 m_{i_{1}}^{2}-4 m_{i_{2}}^{2}\right)+\mathcal{O}(\epsilon) \\
I_{i_{1}}\left[\mu^{2}\right] & =\frac{m_{i_{1}}^{4}}{2}+\mathcal{O}(\epsilon)
\end{aligned}
$$

where $s_{i j}$ were defined in Eq. (41) and $v$ is an arbitrary vector. The tadpole of rank 2 appearing in Eq. (B.2) can also be written as a function of the scalar tadpole integral $I_{i_{1}}$ as follows

$$
I_{i_{1}}\left[\left(\left(q+p_{i_{1}}\right) \cdot e_{3}\right)\left(\left(q+p_{i_{1}}\right) \cdot e_{4}\right)\right]=m_{i_{1}}^{2} \frac{\left(e_{3} \cdot e_{4}\right)}{4}\left(I_{i_{1}}+\frac{m_{i_{1}}^{2}}{2}\right)+\mathcal{O}(\epsilon) .
$$

Since the vector $e_{2}$ in the bubble integral of rank 3 appearing in Eq. (B.2) is massless, the corresponding integral is simply proportional to the form factor $B_{111}$,

$$
I_{i_{1} i_{2}}\left[\left(\left(q+p_{i_{1}}\right) \cdot e_{2}\right)^{3}\right]=\left(\left(p_{i_{2}}-p_{i_{1}}\right) \cdot e_{2}\right)^{3} B_{111}\left(s_{i_{2} i_{1}}, m_{i_{1}}^{2}, m_{i_{2}}^{2}\right) .
$$

The form factor can be computed using the formulas of Ref. [58], as a function of form factors of scalar integrals $B_{0}$. In the special case with $s_{i_{2} i_{1}}=0$ we use Eq. (A.6.2) and (A.6.3) of that reference. For the general case $s_{i_{2} i_{1}} \neq 0$ we implement instead the following formula

$$
\begin{aligned}
B_{111}\left(s_{i_{2} i_{1}}, m_{i_{1}}^{2}, m_{i_{2}}^{2}\right)=\frac{1}{4 s_{i_{2} i_{1}}^{3}\{}\{ & s_{i_{2} i_{1}}\left(m_{i_{1}}^{2} I_{i_{1}}+I_{i_{1}}\left[\mu^{2}\right]-m_{i_{2}}^{2} I_{i_{2}}-I_{i_{2}}\left[\mu^{2}\right]\right. \\
& -4 I_{i_{1} i_{2}}\left[\mu^{2}\left(\left(q+p_{i_{1}}\right) \cdot\left(p_{i_{2}}-p_{i_{1}}\right)\right)\right] \\
& \left.-4 m_{i_{1}}^{2} I_{i_{1} i_{2}}\left[\left(q+p_{i_{1}}\right) \cdot\left(p_{i_{2}}-p_{i_{1}}\right)\right]\right) \\
& \left.+4\left(m_{i_{2}}^{2}-m_{i_{1}}^{2}-s_{i_{2} i_{1}}\right) I_{i_{1} i_{2}}\left[\left(\left(q+p_{i_{1}}\right) \cdot\left(p_{i_{2}}-p_{i_{1}}\right)\right)^{2}\right]\right\} .
\end{aligned}
$$

\section{Appendix C Interfaces to Integral Libraries}

NINJA already implements interfaces for the ONELOOP and the LOOPTOOLS integral libraries. These libraries have been used in a large number of computations and provide very reliable results, hence they should suffice for most purposes. However, NinJA has been designed considering the possibility of using any other library of Master Integrals.

The Master Integrals are computed by calling virtual methods of the abstract class ninja: : IntegralLibrary, which is defined in the header file ninja/integral_library.hh. 
Therefore, any library of Master Integrals can be interfaced by implementing a class derived from IntegralLibrary. Each method of the library corresponds to a different Master Integral appearing in Eq. (15), which should be implemented for both real and complex internal masses (and optionally for the massless case). An implementation of higher-rank integrals can also be provided but it is not needed, since NinJa has a default implementation of them in terms of lower rank integrals. There are two further methods, namely init and exit. The former is called inside the method Amplitude: : evaluate just before the computation of the first needed Master Integral, while the latter is called after the last Master Integral has been computed. The method init

$$
\text { virtual void init(Real muRsq) = } 0 \text {; }
$$

takes as input the square of the renormalization scale to be used in the subsequent calls of the methods implementing the Master Integrals. It can also be used in order to perform any other initialization the library might need before computing the integrals. The exit method instead, does not need to be implemented and by default it will not perform any action. In Section 5.6 we gave an example of a case where a non-trivial implementation of the exit method could be useful.

The other methods should compute the finite part and the poles of the corresponding Master Integrals. As an example, the methods for the box integrals have the following declarations

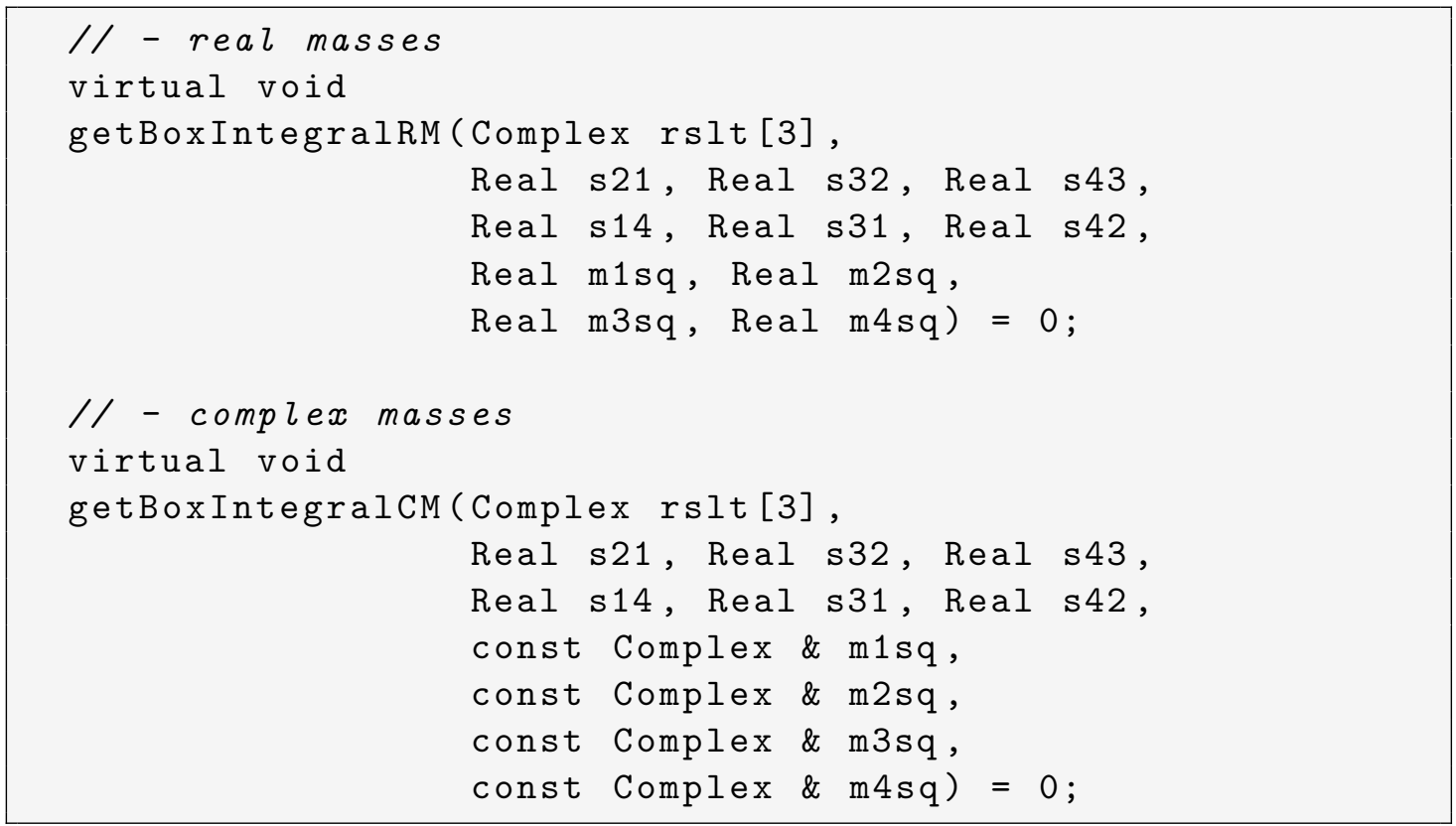

and they must write the $\mathcal{O}\left(\epsilon^{-i}\right)$ term of the result in the $i$-th entry of the array rslt, for $i \in\{0,1,2\}$. The arguments are the invariants $s_{i j}$ and the squared masses $m_{i}^{2}$. Similar methods need to be provided for 3-point, 2-point and 1-point Master Integrals, as described in detail in the comments inside the header file ninja/integral_library.hh.

\section{C.1 Built-in interfaces}

Examples of implementation of this interface for the libraries OnELoop and LoopTools can be found in the source code. More in detail, we define the instances ninja: : avh_olo and 
ninja: : loop_tools of the classes ninja: :AvHOneLoop and ninja: :LoopTools respectively, which implement the methods described above as wrappers of the corresponding routines in each integral library.

The ONELoop interface also implements a cache of Master Integrals on top of these routines. The cache is implemented similarly to a hash table, which allows constant-time look-up of each computed integral from its arguments. Hence, the methods of the AvHOneLoop class will call the routines of the ONELOOP library only if a Master Integral is not found in the cache. The cache can be cleared with the class method AvHOneLoop: : clearIntegralCache. During a phase-space integration, we suggest calling this method once per phase space point, especially for more complex processes. This method does not completely free the allocated memory, but keeps the buckets of the hash table available in order to store the integrals more efficiently in subsequent calls of the respective methods. If the user wishes to completely free the allocated memory, the method AvHOneLoop: : freeIntegralCache can be used, although in general clearIntegralCache should be preferred. As already mentioned, every instance of AvHOneLoop has a cache of Master Integrals as data member. This can be useful for building multi-threaded applications, as discussed in the examples of Section 5.6.

Since LoopTools already has an internal cache of Master Integrals, the implementation of its interface is much simpler and only consists in wrapper of its routines. We implemented a clearIntegralCache method in the LoopTools class as well, which in this case simply calls the routine which clears the cache of integrals in LoopTooLs.

\section{References}

[1] F. Cachazo, P. Svrcek and E. Witten, JHEP 0409 (2004) 006 [hep-th/0403047].

[2] R. Britto, F. Cachazo and B. Feng, Nucl. Phys. B 715 (2005) 499 [hep-th/0412308].

[3] R. Britto, F. Cachazo, B. Feng and E. Witten, Phys. Rev. Lett. 94 (2005) 181602 [hepth/0501052].

[4] Z. Bern, L. J. Dixon, D. C. Dunbar and D. A. Kosower, Nucl. Phys. B 425 (1994) 217 [hep-ph/9403226].

[5] R. Britto, F. Cachazo and B. Feng, Nucl. Phys. B 725 (2005) 275 [hep-th/0412103].

[6] G. Ossola, C. G. Papadopoulos and R. Pittau, Nucl. Phys. B 763 (2007) 147 [hep$\mathrm{ph} / 0609007]$.

[7] R. K. Ellis, W. T. Giele and Z. Kunszt, JHEP 0803 (2008) 003 [arXiv:0708.2398 [hep$\mathrm{ph}]$.

[8] P. Mastrolia and G. Ossola, JHEP 1111 (2011) 014 [arXiv:1107.6041 [hep-ph]].

[9] S. Badger, H. Frellesvig and Y. Zhang, JHEP 1204 (2012) 055 [arXiv:1202.2019 [hep$\mathrm{ph}]$.

[10] Y. Zhang, JHEP 1209 (2012) 042 [arXiv:1205.5707 [hep-ph]].

[11] P. Mastrolia, E. Mirabella, G. Ossola and T. Peraro, Phys. Lett. B 718 (2012) 173 [arXiv:1205.7087 [hep-ph]]. 
[12] P. Mastrolia, E. Mirabella, G. Ossola and T. Peraro, Phys. Lett. B 727 (2013) 532 [arXiv:1307.5832 [hep-ph]].

[13] G. Ossola, C. G. Papadopoulos and R. Pittau, JHEP 0803 (2008) 042 [arXiv:0711.3596 [hep-ph]].

[14] P. Mastrolia, G. Ossola, T. Reiter and F. Tramontano, JHEP 1008 (2010) 080 [arXiv:1006.0710 [hep-ph]].

[15] T. Hahn and M. Perez-Victoria, Comput. Phys. Commun. 118 (1999) 153 [hep$\mathrm{ph} / 9807565]$.

[16] A. van Hameren, C. G. Papadopoulos and R. Pittau, JHEP 0909 (2009) 106 [arXiv:0903.4665 [hep-ph]].

[17] G. Bevilacqua, M. Czakon, M. V. Garzelli, A. van Hameren, A. Kardos, C. G. Papadopoulos, R. Pittau and M. Worek, Comput. Phys. Commun. 184 (2013) 986 [arXiv:1110.1499 [hep-ph]].

[18] C. F. Berger, Z. Bern, L. J. Dixon, F. Febres Cordero, D. Forde, H. Ita, D. A. Kosower and D. Maitre, Phys. Rev. D 78 (2008) 036003 [arXiv:0803.4180 [hep-ph]].

[19] V. Hirschi, R. Frederix, S. Frixione, M. V. Garzelli, F. Maltoni and R. Pittau, JHEP 1105 (2011) 044 [arXiv:1103.0621 [hep-ph]].

[20] G. Cullen, N. Greiner, G. Heinrich, G. Luisoni, P. Mastrolia, G. Ossola, T. Reiter and F. Tramontano, Eur. Phys. J. C 72 (2012) 1889 [arXiv:1111.2034 [hep-ph]].

[21] F. Cascioli, P. Maierhofer and S. Pozzorini, Phys. Rev. Lett. 108 (2012) 111601 [arXiv:1111.5206 [hep-ph]].

[22] S. Badger, B. Biedermann and P. Uwer, Comput. Phys. Commun. 182 (2011) 1674 [arXiv:1011.2900 [hep-ph]].

[23] S. Badger, B. Biedermann, P. Uwer and V. Yundin, Comput. Phys. Commun. 184 (2013) 1981 [arXiv:1209.0100 [hep-ph]].

[24] P. Mastrolia, E. Mirabella and T. Peraro, JHEP 1206 (2012) 095 [Erratum-ibid. 1211 (2012) 128] [arXiv:1203.0291 [hep-ph]].

[25] A. van Hameren, Comput. Phys. Commun. 182 (2011) 2427 [arXiv:1007.4716 [hep-ph]].

[26] H. van Deurzen, G. Luisoni, P. Mastrolia, E. Mirabella, G. Ossola and T. Peraro, Phys. Rev. Lett. 111 (2013) 171801 [arXiv:1307.8437 [hep-ph]].

[27] H. van Deurzen, G. Luisoni, P. Mastrolia, E. Mirabella, G. Ossola and T. Peraro, arXiv:1312.6678 [hep-ph].

[28] J. A. M. Vermaseren, math-ph/0010025.

[29] J. Kuipers, T. Ueda, J. A. M. Vermaseren and J. Vollinga, Comput. Phys. Commun. 184 (2013) 1453 [arXiv:1203.6543 [cs.SC]]. 
[30] J. Kuipers, T. Ueda and J. A. M. Vermaseren, arXiv:1310.7007 [cs.SC].

[31] R. K. Ellis and G. Zanderighi, JHEP 0802 (2008) 002 [arXiv:0712.1851 [hep-ph]].

[32] D. Forde, Phys. Rev. D 75 (2007) 125019 [arXiv:0704.1835 [hep-ph]].

[33] F. del Aguila and R. Pittau, JHEP 0407 (2004) 017 [hep-ph/0404120].

[34] P. Mastrolia, G. Ossola, C. G. Papadopoulos and R. Pittau, JHEP 0806 (2008) 030 [arXiv:0803.3964 [hep-ph]].

[35] S. D. Badger, JHEP 0901 (2009) 049 [arXiv:0806.4600 [hep-ph]].

[36] R. Kleiss, W. J. Stirling and S. D. Ellis, Comput. Phys. Commun. 40 (1986) 359.

[37] G. Ossola, C. G. Papadopoulos and R. Pittau, JHEP 0707 (2007) 085 [arXiv:0704.1271 [hep-ph]].

[38] G. J. Gounaris, P. I. Porfyriadis and F. M. Renard, Eur. Phys. J. C 9 (1999) 673 [hep$\mathrm{ph} / 9902230]$.

[39] C. Bernicot, arXiv:0804.0749 [hep-ph].

[40] G. Cullen, M. Koch-Janusz and T. Reiter, Comput. Phys. Commun. 182 (2011) 2368 [arXiv:1008.0803 [hep-ph]].

[41] G. Mahlon, Phys. Rev. D 49 (1994) 2197 [hep-ph/9311213].

[42] Z. Nagy and D. E. Soper, Phys. Rev. D 74 (2006) 093006 [hep-ph/0610028].

[43] T. Binoth, G. Heinrich, T. Gehrmann and P. Mastrolia, Phys. Lett. B 649 (2007) 422 [hep-ph/0703311].

[44] W. Gong, Z. Nagy and D. E. Soper, Phys. Rev. D 79 (2009) 033005 [arXiv:0812.3686 [hep-ph]].

[45] C. Bernicot and J. -P. .Guillet, JHEP 0801 (2008) 059 [arXiv:0711.4713 [hep-ph]].

[46] C. Bernicot, arXiv:0804.1315 [hep-ph].

[47] W. Beenakker, S. Dittmaier, M. Kramer, B. Plumper, M. Spira and P. M. Zerwas, Phys. Rev. Lett. 87 (2001) 201805 [hep-ph/0107081].

[48] W. Beenakker, S. Dittmaier, M. Kramer, B. Plumper, M. Spira and P. M. Zerwas, Nucl. Phys. B 653 (2003) 151 [hep-ph/0211352].

[49] S. Dawson, L. H. Orr, L. Reina and D. Wackeroth, Phys. Rev. D 67 (2003) 071503 [hep-ph/0211438].

[50] S. Dawson, C. Jackson, L. H. Orr, L. Reina and D. Wackeroth, Phys. Rev. D 68 (2003) 034022 [hep-ph/0305087].

[51] S. Dittmaier, M. Kramer, 1 and M. Spira, Phys. Rev. D 70 (2004) 074010 [hep$\mathrm{ph} / 0309204]$. 
[52] H. van Deurzen, N. Greiner, G. Luisoni, P. Mastrolia, E. Mirabella, G. Ossola, T. Peraro and J. F. von Soden-Fraunhofen et al., Phys. Lett. B 721 (2013) 74 [arXiv:1301.0493 [hep-ph]].

[53] G. Cullen, H. van Deurzen, N. Greiner, G. Luisoni, P. Mastrolia, E. Mirabella, G. Ossola and T. Peraro et al., Phys. Rev. Lett. 111 (2013) 131801 [arXiv:1307.4737 [hep-ph]].

[54] H. van Deurzen, Acta Phys. Polon. B 44 (2013) 11, 2223.

[55] T. Binoth, J. -P. .Guillet, G. Heinrich, E. Pilon and T. Reiter, Comput. Phys. Commun. 180 (2009) 2317 [arXiv:0810.0992 [hep-ph]].

[56] G. Cullen, J. P. .Guillet, G. Heinrich, T. Kleinschmidt, E. Pilon, T. Reiter and M. Rodgers, Comput. Phys. Commun. 182 (2011) 2276 [arXiv:1101.5595 [hep-ph]].

[57] J. P. .Guillet, G. Heinrich and J. F. von Soden-Fraunhofen, arXiv:1312.3887 [hep-ph].

[58] R. G. Stuart, Comput. Phys. Commun. 48 (1988) 367. 